\title{
A COMPONENTWISE CONVEX SPLITTING SCHEME FOR DIFFUSE INTERFACE MODELS WITH VAN DER WAALS AND PENG-ROBINSON EQUATIONS OF STATE*
}

\author{
XIAOLIN FAN ${ }^{\dagger}$, JISHENG KOU ${ }^{\ddagger}$, ZHONGHUA QIAO ${ }^{\S}$, AND SHUYU SUN ${ }^{\dagger}$
}

\begin{abstract}
This paper presents a componentwise convex splitting scheme for numerical simulation of multicomponent two-phase fluid mixtures in a closed system at constant temperature, which is modeled by a diffuse interface model equipped with the Van der Waals and the Peng-Robinson equations of state (EoS). The Van der Waals EoS has a rigorous foundation in physics, while the Peng-Robinson EoS is more accurate for hydrocarbon mixtures. First, the phase field theory of thermodynamics and variational calculus are applied to a functional minimization problem of the total Helmholtz free energy. Mass conservation constraints are enforced through Lagrange multipliers. A system of chemical equilibrium equations is obtained which is a set of second-order elliptic equations with extremely strong nonlinear source terms. The steady state equations are transformed into a transient system as a numerical strategy on which the scheme is based. The proposed numerical algorithm avoids the indefiniteness of the Hessian matrix arising from the second-order derivative of homogeneous contribution of total Helmholtz free energy; it is also very efficient. This scheme is unconditionally componentwise energy stable and naturally results in unconditional stability for the Van der Waals model. For the Peng-Robinson EoS, it is unconditionally stable through introducing a physics-preserving correction term, which is analogous to the attractive term in the Van der Waals EoS. An efficient numerical algorithm is provided to compute the coefficient in the correction term. Finally, some numerical examples are illustrated to verify the theoretical results and efficiency of the established algorithms. The numerical results match well with laboratory data.
\end{abstract}

Key words. convex splitting, sequential splitting, equation of state, Van der Waals EoS, PengRobinson EoS, multicomponent two-phase systems

AMS subject classifications. 65N30, 65N50, 49S05

DOI. $10.1137 / 16 \mathrm{M} 1061552$

1. Introduction. Multiphase multicomponent fluid systems are key building blocks in many engineering and science fields in which numerous research has been done, especially in reservoir engineering [7, 29,32] and environmental protection such as carbon dioxide capturing and sequestration [22, 27]. Though plenty of research work has been done on these issues $[8,11,19,26,30,31,33]$, there is still a great deal of challenge on the understanding of gas bubbles, liquid droplets, interfaces between gas-liquid phases, and capillary pressure taking place in the multiphase fluid systems.

*Submitted to the journal's Computational Methods in Science and Engineering section February 16, 2016; accepted for publication (in revised form) September 26, 2016; published electronically January 19, 2017. A small portion of the findings reported in this article were originally presented in the ICCS (International Conference on Computational Science) proceedings in June, 2016 [10].

http://www.siam.org/journals/sisc/39-1/M106155.html

Funding: The work of the first and fourth authors was supported by funding from King Abdullah University of Science and Technology (KAUST). The work of the second author was partially supported by National Natural Science Foundation of China (11301163). The work of the third author was partially supported by Hong Kong Research Grant Council GRF grants 509213, 15302214 and NSFC/RGC joint research scheme N_HKBU204/12.

${ }^{\dagger}$ Physical Science and Engineering (PSE), King Abdullah University of Science and Technology (KAUST), Thuwal 23955-6900, Saudi Arabia (Xiaolin.Fan.1@kaust.edu.sa, Shuyu.Sun@kaust.edu.sa).

${ }^{\ddagger}$ School of Mathematics and Statistics, Hubei Engineering University, Xiaogan 432000, Hubei, China (koujisheng@163.com).

$\S$ Department of Applied Mathematics, The Hong Kong Polytechnic University, Hung Hom, Hong Kong (zhonghua.qiao@polyu.edu.hk). 
These all rely on modeling and simulating correctly the physics of multiphase fluid systems. The most critical aspect is the phenomena occurring in the interfaces $[17$, $18,20,24]$.

Currently, as one of three most popular methodologies to model multiphase interfaces (including molecular simulation, sharp interface modeling, diffuse interface modeling [3]), diffuse interface modeling (or phase field modeling or gradient theory) has attracted the attention of many scientists and engineers, for example, as reported in the literature $[2,13,25,28,34]$, to name but a few. In these works, a simple double-well potential is adopted, which has been qualitatively (instead of quantitatively) analyzed; this is inadequate for engineering practice. When a realistic model (take the Peng-Robinson model [23] as an example) is applied for hydrocarbon mixtures, the interfaces can be modeled quantitatively [24]. In this setting, however, the diffuse interface modeling becomes much more complicated and more challenging for developing efficient, easy-to-implement, energy stable numerical schemes.

There has been some work on numerical simulation of multicomponent phase field models. In the work by Kim, Kang, and Lowengrub [14], the authors proposed a conservative, second-order accurate fully implicit discretization scheme for the three-phase Cahn-Hilliard systems. Boyer and Minjeaud in [4] developed an energydecaying scheme for a three-component Cahn-Hilliard model. The quartic free energy is adopted in their work. In our work, we take advantage of the Helmholtz free energy based on physical models, i.e., the Van der Waals equation of state (EoS) and more realistic one, i.e., the Peng-Robinson EoS. In particular, the Peng-Robinson $\mathrm{EoS}$ is most widely used in subsurface flow such as petroleum engineering modeling and subsurface $\mathrm{CO}_{2}$ sequestration [22]. These models are generally applied for multicomponent systems. Both models are more complicated than the quartic free energy model, and it is challenging to develop some physics-preserving (for example, energy decaying) numerical schemes.

In the work by Qiao and Sun [24], the diffuse interface model with the PengRobinson expression of Helmholtz free energy density was proposed to model the interfaces in two-phase hydrocarbon systems. With the Peng-Robinson expression of the Helmholtz free energy density, the model becomes very complicated even for single-component systems. The authors there designed an efficient scheme for singlecomponent systems. They established a clean convex splitting of the total Helmholtz free energy and treated the convex and concave parts separately, which stabilizes the algorithm. The method of convex splitting of energy functional was first proposed by Eyre [9] and has been widely investigated and has obtained lots of success, in particular for gradient flow systems. This strategy satisfies certain physics-preserving properties including conservation of mass and the decaying of free energy. The energy decaying property is particularly critical, but it cannot be guaranteed with fully explicit and even fully implicit methods. Though this convex splitting algorithm is unconditionally stable and fast converging, it has not been systematically applied to multicomponent multiphase fluid systems because of its challenges arising from the indefiniteness of the Hessian matrix.

When multicomponent systems are considered, in theory it is natural to extend the energy decaying algorithm with convex splitting. In practice, however, it is not straightforward to extend the convex splitting from single-component to multicomponent systems. The convex splitting strategy cannot be utilized for all components simultaneously in a straightforward manner due to cross interactions among species. In particular, the Hessian matrices for $f_{0}^{b}$ and $f_{0}^{a}$ (which will be given in subsequent sections) might be indefinite, which can be shown at least for the case of binary 
mixture systems with either the Van der Waals or the Peng-Robinson model. The indefiniteness of the Hessian matrices is likely to be true for a mixture of three and more components. Convex splitting of multivariable systems usually can be achieved by two general approaches; one is through matrix splitting and the other is sequentially componentwise splitting. In this work, we focus on the latter method.

In the work, we first convert a system of chemical equilibrium equations which is equivalent to the original minimization problem (that will be provided in the succeeding sections) into a time-dependent version by adding a time-derivative term into each equation. A componentwise convex splitting time-marching scheme is considered on the transient partial differential equations, whose solution converges to that of corresponding steady state partial differential equations.

For the transient equations, we iterate for all components at each time step. For each chosen component, we treat the corresponding equation using semi-implicit time discretization through convex splitting, with all other components taking the previous time-step values. This sequential convex splitting strategy maintains the property of energy decaying in a componentwise way and naturally leads to the unconditional energy stability for each time step. This method works well even with the indefiniteness of relevant Hessian matrices. Since this method is carried out sequentially on components, it improves numerical efficiency and reduces the requirement on memory storage when dealing with a large number of components. For the time-discrete system, we adopt the Raviart-Thomas (RT) [6] mixed finite element method (which has the appealing properties of local mass conservation and the same order accuracy for both flux and scalar variables, and has the capability of treating general geometry) to carry out the spatial discretization. The Newton method is employed to solve the fully discretized nonlinear equations.

Two types of equations of state (i.e., the Van der Waals and Peng-Robinson EoS) are considered for the Helmholtz free energy instead of the double-well potential. When the Van der Waals model gets involved, the convex splitting method can be utilized for the mixture of hydrocarbon species, which has better quantitative modeling behaviors than that of the double-well model. The Van der Waals model is here mainly used for theoretical analysis of our algorithms and paves the way for the correction term in the Peng-Robinson model, which is more realistic for hydrocarbon mixtures. However, when the Peng-Robinson expression of Helmholtz free energy is applied, the attraction term becomes quite complicated and brings some difficulty in establishing numerical schemes. A physics-preserving adjustment term is borrowed from the Van der Waals model to carry out the desired splitting. Having this correction term, the free energy can be split into summation of convex and concave parts in a componentwise fashion and the component-wise convex splitting scheme is proposed accordingly.

The rest of this paper is structured as follows. In section 2 , the mathematical statement of the problem is addressed; it consists of the minimization of total Helmholtz free energy corresponding to the original description of the problem, converting the minimization functional into an equivalent system of partial differential equations (chemical equilibrium equations). We review the foundations of thermodynamics theory at equilibrium state, and a few formulas for the Van der Waals and the Peng-Robinson expressions of Helmholtz free energy and influence parameters for describing interface properties. In section 3, the chemical equilibrium equations are first transformed into transient partial differential equations by adding time-derivative terms. A componentwise, time-marching procedure and the RT mixed finite element method are established to approximate the time-dependent equations spatially. In 
section 4, some numerical examples are demonstrated to verify the established algorithm; the results are shown and are compared with laboratory data. The conclusion is drawn in section 5 .

\section{Diffuse interface models for multiphase multicomponent systems.}

2.1. The problem statement for an equilibrium state. We consider a multiphase multicomponent fluid system comprised of $M$ components in a container of invariant volume occupying a domain denoted by $\Omega \subset \mathbb{R}^{d}(d=1,2,3)$ at constant temperature $T$. For simplicity of analysis, the gravity is neglected in the current manuscript. Denote by $n_{i}$ the molar density of the $i$ th species. Let

$$
\mathbf{n}(\mathbf{x})=\left(n_{1}(\mathbf{x}), \ldots, n_{M}(\mathbf{x})\right)^{T}=\frac{\left(d N_{1}, \ldots, d N_{M}\right) T}{d V}
$$

represent the molar densities of all species, and let $n(\mathbf{x})=n_{1}(\mathbf{x})+\cdots+n_{M}(\mathbf{x})$. Here $d N_{i}$ is the molar quantity of species $i$ in $d V$, and $d V$ is an elementary volume at position $\mathbf{x}$.

Based on the gradient theory for multiphase systems, the total Helmholtz free energy is composed of two parts: $F_{0}$ being the thermodynamics theory of homogeneous fluids and $F_{\nabla}$ the gradient part of inhomogeneity. So the total Helmholtz free energy of the system is given by

$$
\begin{aligned}
F(\mathbf{n}) & =F(\mathbf{n} ; T, \Omega)=F_{0}(\mathbf{n} ; T, \Omega)+F_{\nabla}(\mathbf{n} ; T, \Omega) \\
& =\int_{\Omega} f(\mathbf{n} ; T) d \mathbf{x}=\int_{\Omega} f_{0}(\mathbf{n} ; T) d \mathbf{x}+\int_{\Omega} f_{\nabla}(\mathbf{n} ; T) d \mathbf{x} .
\end{aligned}
$$

In the above integral, $f(\mathbf{n} ; T), f_{0}(\mathbf{n} ; T)$, and $f_{\nabla}(\mathbf{n} ; T)$ stand for the total Helmholtz free energy density, the Helmholtz free energy density of homogeneous fluids, and the Helmholtz free energy density from gradient contribution, respectively. The homogeneous part $f_{0}(\mathbf{n})=f_{0}(\mathbf{n} ; T)$ is given by the traditional thermodynamic formula which will be provided with two specific EoS, the Van der Waals EoS and the PengRobinson EoS in the subsequent sections. The inhomogeneous part $f_{\nabla}(\mathbf{n})=f_{\nabla}(\mathbf{n} ; T)$ is computed by

$$
f_{\nabla}(\mathbf{n})=\frac{1}{2} \sum_{i=1}^{M} \sum_{j=1}^{M} c_{i j} \nabla n_{i} \cdot \nabla n_{j}
$$

in which the coefficient $c_{i j}$ denotes the influence parameter. $c_{i j}$ theoretically depends on temperature $T$, and molar concentrations will be given in the subsequent sections.

In this work, only the equilibrium state of the mixture is considered. In light of thermodynamics theory, the total Helmholtz free energy is minimized at equilibrium state in a closed system with fixed domain and constant temperature. The mathematical description of the problem can be stated as follows: to seek $\mathbf{n} \in H$ such that

$$
F(\mathbf{n})=\min _{\widehat{\mathbf{n}} \in H} F(\widehat{\mathbf{n}})
$$

subject to

$$
\int_{\Omega} n_{i} d \mathbf{x}=N_{i}, \quad i=1, \ldots, M
$$

where $H$ is a function space satisfying certain regularity. Equation (2.3) describes that the total mass of each component of the mixture is constant under the assumed conditions. 
2.2. Necessary conditions for the phase equilibrium state. According to the thermodynamics theory, at equilibrium, the total chemical potential is uniformly invariant in the whole domain for each species, which is mathematically expressed as

$$
\mu_{i}(\mathbf{x})=U_{i} \forall \mathbf{x} \in \Omega, \quad i=1, \ldots, M,
$$

where $U_{i} \in \mathbb{R}$ is constant. Here the total chemical potential consists of two parts, homogeneous fluids and the inhomogeneity contribution, so

$$
\mu_{i}(\mathbf{x})=\mu_{0, i}(\mathbf{x})+\mu_{\nabla, i}(\mathbf{x}) \forall \mathbf{x} \in \Omega, \quad i=1, \ldots, M .
$$

By thermodynamics theory, the chemical potential of homogeneous term is defined as

$$
\mu_{0, i}=\left(\frac{\partial F_{0}}{\partial N_{i}}\right)_{T, V, \mathbf{N}_{\neq i}}=\left(\frac{\partial f_{0}}{\partial n_{i}}\right)_{T, \mathbf{n}_{\neq i}}, \quad i=1, \ldots, M,
$$

where $\mathbf{N}_{\neq i}$ and $\mathbf{n}_{\neq i}$ denote the vectors $\left(N_{1}, \ldots, N_{i-1}, N_{i+1}, \ldots, N_{M}\right)$ and $\left(n_{1}, \ldots\right.$, $\left.n_{i-1}, n_{i+1}, \ldots, n_{M}\right)$, respectively. On the basis of the variational calculus, the inhomogeneous part can be computed by

$$
\mu_{\nabla, i}=\frac{\partial f_{\nabla}(\mathbf{n})}{\partial n_{i}}=\frac{1}{2} \sum_{j=1}^{M} \sum_{k=1}^{M} \frac{\partial c_{k j}}{\partial n_{i}} \nabla n_{k} \cdot \nabla n_{j}-\sum_{j=1}^{M} \nabla \cdot\left(c_{i j} \nabla n_{j}\right), i=1, \ldots, M
$$

Frequently, the influence parameter $c_{i j}$ is assumed to be independent of the molar densities of all species, and hence (2.7) is simplified as

$$
\mu_{\nabla, i}=-\sum_{j=1}^{M} c_{i j} \Delta n_{j}, \quad i=1, \ldots, M .
$$

Substituting (2.6) and (2.8) into (2.5), we finally have

$$
-\sum_{j=1}^{M} c_{i j} \Delta n_{j}=\mu_{i}-\mu_{0, i}(\mathbf{n}), \quad i=1, \ldots, M
$$

The system of (2.9) indicates the chemical equilibrium conditions under the above assumption of $c_{i j}$. Using the methods of Lagrange multipliers and variational calculus for the original system of (2.2) and (2.3) can lead to the same equations of (2.9); see [15].

Equation (2.9) is a system of tightly coupled second-order elliptic partial differential equations with strongly nonlinear source terms $\mu_{0, i}(\mathbf{n})$ due to the strong nonlinearity of $f_{0}$, which will be given in subsequent sections. This strong nonlinearity and very small magnitude values of $c_{i j}$ yield substantial difficulty for developing numerical algorithms. To solve this system of equations efficiently and physically satisfactorily, it is important to establish an efficient numerical scheme for two-phase multicomponent fluid systems.

2.3. Bulk properties of mixtures modeled by the Van der Waals EoS. The Van der Waals EoS theoretically approximates the behavior of real fluids to some extent, which was originally derived in statistical molecular thermodynamics [12]. Even though it is still quite idealized for a real fluid, the Van der Waals fluid is often used for theoretical analysis and numerical testing due to its simplicity, elegance, and 
physical foundation. It also plays a more important role in the correction term that we will borrow for efficient numerical solution of the Peng-Robinson model. We now briefly describe the relevant quantities based on this EoS in this section.

The Helmholtz free energy density $f_{0}(\mathbf{n})$ is written as summation of two terms, the ideal part and the excess one, so it is computed by

$$
f_{0}(\mathbf{n})=f_{0}^{\text {ideal }}(\mathbf{n})+f_{0}^{\text {excess }}(\mathbf{n})
$$

in which the ideal part is given by

$$
f_{0}^{\text {ideal }}(\mathbf{n})=R T \sum_{i=1}^{M} n_{i}\left(\ln n_{i}-1\right),
$$

and the excess term by

$$
f_{0}^{\text {excess }}(\mathbf{n})=-R T n \ln (1-b n)-a n^{2} .
$$

$T$ and $R$ throughout this manuscript denote the temperature of the fluids and the universal gas constant, respectively. The parameter $a$ is related to interparticle attractive force between a couple of particles, and $b$ is associated with the size of each particle. Parameters $a$ and $b$ and the derivation of the Van der Waals EoS from the free energy point of view are given in Appendix A.

2.4. Bulk properties of mixtures modeled by the Peng-Robinson EoS. The Peng-Robinson EoS is modified on the basis of the Van der Waals EoS. It is widely applied in engineering fields, for instance, petroleum engineering. The PengRobinson EoS models the behavior of real fluids more accurately, in particular for hydrocarbon mixtures, but is more complicated than the Van der Waals model. We describe some quantities in brief below. The Peng-Robinson expression of Helmholtz free energy density of the homogeneous fluid has the same first two terms as those in the Van der Waals form; the only formal difference from the Van der Waals expression is the last term. The total free energy density for the Peng-Robinson EoS reads as follows:

$$
\begin{aligned}
f_{0}(\mathbf{n})= & R T \sum_{i=1}^{M} n_{i}\left(\ln n_{i}-1\right)-R T n \ln (1-b n) \\
& +\frac{a(T) n}{2 \sqrt{2} b} \ln \left(\frac{1+(1-\sqrt{2}) b n}{1+(1+\sqrt{2}) b n}\right) .
\end{aligned}
$$

Parameters $a$ and $b$ and the EoS for the Peng-Robinson model are given in Appendix B.

2.5. Influence parameters computed by geometric mean. The influence parameter $c_{i j}$ characterizes phase interface properties of a pair of species $i$ and $j$ in the fluid mixture. It is obtained by using mixing rules. One of the most used rules is the modified geometric mean rule which is given by

$$
c_{i j}=\left(1-\beta_{i j}\right) \sqrt{c_{i} c_{j}}, \quad i, j=1, \ldots, M,
$$

where $\beta_{i j}$ represents the binary interaction coefficient for the influence parameter. Among these, engineering practice suggests that the geometric mean mixing rule is 
most popularly adopted, that is, when $\beta_{i j}=0$ (see $[16,21]$ ). We apply this rule in the current manuscript. In the equation, $c_{i}$ is the influence parameter of a single species computed by (see $[5,21])$

$$
c_{i}=a_{i} b_{i}^{\frac{2}{3}}\left(m_{1, i}\left(1-\frac{T}{T_{c i}}\right)+m_{2, i}\right), \quad i=1, \ldots, M,
$$

where $m_{1, i}$ and $m_{2, i}$ are correlated by the acentric factor $\omega_{i}$ :

$$
m_{1, i}=-\frac{10^{-16}}{1.2326+1.2757 \omega_{i}}, \quad m_{2, i}=-\frac{10^{-16}}{0.9051+1.5410 \omega_{i}}, \quad i=1, \ldots, M .
$$

3. Numerical solutions of chemical equilibrium equations. In this section we will establish an efficient numerical algorithm for the system of the chemical equilibrium equations (2.9) which is equivalent to (2.2). The aforementioned strong nonlinearity in the source terms of (2.9) and the extremely small values of $c_{i j}$ give rise to a great challenge of the numerical solution. In the iterative methods utilized to solve the algebraic system arising from the spatial discretization of the partial differential equations, efficiency is a major concern; stability is even trickier to deal with. We adopt the approach proposed by Qiao and Sun [24] to utilize the corresponding transient problems for constructing an efficient and stable iterative scheme for (2.9).

3.1. The transient version of chemical equilibrium equations. For the sake of concision, we let $\mathbf{n}=\mathbf{n}(\mathbf{x}, t), n_{i}=n_{i}(\mathbf{x}, t)$ and abbreviate relevant notations without confusion in the succeeding sections. Adding a corresponding time-derivative term $\frac{\partial n_{i}}{\partial t}$ into the left-hand side of the $i$ th equation of (2.9), we get

$$
\frac{\partial n_{i}}{\partial t}-\sum_{j=1}^{M} c_{i j} \Delta n_{j}=\mu_{i}-\mu_{0, i}(\mathbf{n}(\mathbf{x}, t)), \quad i=1, \ldots, M .
$$

The above system of partial differential equations can be uniquely solved with proper initial conditions and boundary conditions as well as a set of specified bulk chemical potentials $\mu_{i}$.

Although (3.1) can be solved to determine a unique time-dependent solution, the solution is quite sensitive to the parameters $\mu_{i}$ according to numerical tests. Moreover, this system of partial differential equations is not mass conservative even if the zero Neumann boundary condition is imposed. In most realistic situations, this nonconservation will lead to the final steady state solution being single phase, which is not what we expect.

To fix the aforementioned two issues, Lagrange multipliers $\mu_{i}(t)$ are introduced for enforcing the mass conservation. That is, $\mu_{i}$ are considered as unknown parameters that depend on time but are spatially constant. As time goes infinite, $\mu_{i}(t)$ approximates $\mu_{i}$, and therefore (3.1) becomes

$$
\frac{\partial n_{i}}{\partial t}-\sum_{j=1}^{M} c_{i j} \Delta n_{j}=\mu_{i}(t)-\mu_{0, i}(\mathbf{n}(\mathbf{x}, t)), \quad i=1, \ldots, M .
$$

The mass conservation equations accordingly are changed into

$$
\int_{\Omega} n_{i}(\mathbf{x}, t) d \mathbf{x}=N_{i}, \quad i=1, \ldots, M .
$$

Copyright $@$ by SIAM. Unauthorized reproduction of this article is prohibited. 
To close the above system, appropriate initial and boundary conditions are required. Here we adopt the zero Neumann boundary condition which indicates the normal derivative of molar density for each component. The initial condition is provided to give the total mass amount of each species in the closed system; apparently the total mass of a single component is constant. The initial and zero Neumann boundary conditions are written as

$$
\begin{aligned}
\nabla n_{i} \cdot \nu_{\partial \Omega} & =0, \quad i=1, \ldots, M, \\
n_{i}=n_{i}^{\text {init }}, \quad t=0, & i=1, \ldots, M,
\end{aligned}
$$

where $\nu_{\partial \Omega}$ is the outward normal to the domain boundary $\partial \Omega$. The system of (3.2)(3.5) uniquely determines the solution $n_{i}(\mathbf{x}, t)$ and $\mu_{i}(t)$. This time-dependent partial differential equations system has certain physical properties described in the following lemma.

3.1.1. Energy identity. The total Helmholtz free energy of the above system is dissipated with time, which is mathematically stated in the following lemma. Here the temperature of the system is assumed to be constant.

Lemma 3.1. Provided $\mathbf{n}$ is the solution of (3.2)-(3.5), the total Helmholtz free energy reduces with time and satisfies

$$
\frac{d F(\mathbf{n})}{d t}=-\sum_{i=1}^{M}\left\|\frac{\partial n_{i}}{\partial t}\right\|_{L^{2}}^{2} .
$$

Proof. Recall the definition of the total Helmholtz free energy

$$
F(\mathbf{n})=\int_{\Omega} f(\mathbf{n}) d \mathbf{x}=\int_{\Omega}\left(f_{0}(\mathbf{n})+\frac{1}{2} \sum_{i, j=1}^{M} c_{i j} \nabla n_{i} \cdot \nabla n_{j}\right) d \mathbf{x} .
$$

Differentiating with respect to $t$ to $F(\mathbf{n})$ and by integration by parts, we obtain

$$
\begin{aligned}
\frac{d F(\mathbf{n})}{d t} & =\int_{\Omega} \sum_{i=1}^{M}\left(\frac{\partial f_{0}(\mathbf{n})}{\partial n_{i}}-\sum_{j=1}^{M} c_{i j} \Delta n_{j}\right) \frac{\partial n_{i}}{\partial t} d x+\int_{\partial \Omega} \sum_{i=1}^{M} \sum_{j=1}^{M} c_{i j} \frac{\partial n_{i}}{\partial t} \nabla n_{j} \cdot \nu_{\partial \Omega} d s \\
& =\int_{\Omega} \sum_{i=1}^{M}\left(\mu_{0, i}-\sum_{j=1}^{M} c_{i j} \Delta n_{j}\right) \frac{\partial n_{i}}{\partial t} d x+\int_{\partial \Omega} \sum_{i=1}^{M} \sum_{j=1}^{M} c_{i j} \frac{\partial n_{i}}{\partial t} \nabla n_{j} \cdot \nu_{\partial \Omega} d s .
\end{aligned}
$$

So with (3.4) and (3.2)

$$
\frac{d F(\mathbf{n})}{d t}=\int_{\Omega} \sum_{i=1}^{M} \frac{\partial n_{i}}{\partial t}\left(\mu_{i}-\frac{\partial n_{i}}{\partial t}\right) d \mathbf{x} .
$$

By (3.3) and the fact that $\mu_{i}(t)$ is spatially invariant, we get

$$
\int_{\Omega} \mu_{i} \frac{\partial n_{i}}{\partial t} d x=0, \quad i=1, \ldots, M
$$

and therefore we can conclude that

$$
\frac{d F(\mathbf{n})}{d t}=-\int_{\Omega} \sum_{i=1}^{M} \frac{\partial n_{i}}{\partial t} \frac{\partial n_{i}}{\partial t} d x=-\sum_{i=1}^{M}\left\|\frac{\partial n_{i}}{\partial t}\right\|_{L^{2}}^{2} .
$$

This ends the proof. 
3.2. An efficient energy-stable scheme of the transient chemical equilibrium equations with the Van der Waals and the Peng-Robinson expressions of $f_{0}$.

3.2.1. The componentwise convex splitting of $f_{0}$ with the Van der Waals model. In this section, we show that the Helmholtz free energy density with the Van der Waals model has convex and concave parts componentwise.

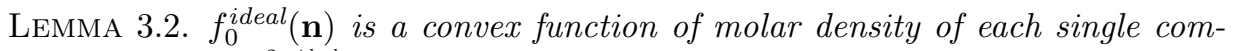
ponent, saying that $\frac{\partial^{2} f_{0}^{i d e a l}}{\partial n_{i}^{2}}>0$.

Proof. Recall that $f_{0}^{\text {ideal }}=R T \sum_{i=1}^{M} n_{i}\left(\ln n_{i}-1\right)$, so we have

$$
\frac{\partial f_{0}^{\text {ideal }}}{\partial n_{i}}=R T \ln n_{i}, \quad \frac{\partial^{2} f_{0}^{\text {ideal }}}{\partial n_{i}^{2}}=R T \frac{1}{n_{i}}>0 .
$$

This completes the proof.

Recall that the excess Helmholtz free energy density of the Van der Waals expression consists of two parts:

$$
\begin{array}{r}
f_{0}^{a}=-a n^{2}, \\
f_{0}^{b}=-R T n \ln (1-b n) .
\end{array}
$$

According to the Van der Waals theory, $f_{0}^{b}$ takes into account of the volume exclusion of the particles, while $f_{0}^{a}$ results from the attraction force of molecules in the fluids. This physical property has rigorous mathematical description in the following two lemmas.

Lemma 3.3. The Helmholtz free energy density from the repulsion force of the Van der Waals fluids is a convex function of the molar density of each component; otherwise stated, $\frac{\partial^{2} f_{0}^{b}}{\partial n_{i}^{2}}>0$.

Proof. Recall that $f_{0}^{b}=-R T n \ln (1-b n)$, which gives

$$
\begin{aligned}
\frac{\partial f_{0}^{b}}{\partial n_{i}} & =-R T\left(\ln (1-b n)-\frac{n b_{i}}{1-b n}\right), \\
\frac{\partial^{2} f_{0}^{b}}{\partial n_{i}^{2}} & =R T\left(\frac{2 b_{i}}{1-b n}+\frac{b_{i}^{2} n}{(1-b n)^{2}}\right) .
\end{aligned}
$$

Since $(1-b n)>0$ according to the physical meaning of $(1-b n)$ (based on Van der Waals theory the total volume occupied by the species is always larger than the volume excluded by the species), $b_{i}>0$, and $n>0$, we have $\frac{\partial^{2} f_{0}^{b}}{\partial n_{i}^{2}}>0$.

Lemma 3.4. The Helmholtz free energy density from the attraction force of Van der Waals fluids is a concave function of molar density of every single component; in other words, $\frac{\partial^{2} f_{0}^{a}}{\partial n_{i}^{2}}<0$.

Proof. The first-order partial derivative of $f_{0}^{a}$ with $n_{i}$ gives

$$
\frac{\partial f_{0}^{a}}{\partial n_{i}}=-2 \sum_{j=1}^{M} a_{i j} n_{j} .
$$

Copyright $\odot$ by SIAM. Unauthorized reproduction of this article is prohibited. 
And so the second-order partial derivative of $f_{0}^{a}$ with $n_{i}$ is

$$
\frac{\partial^{2} f_{0}^{a}}{\partial n_{i}^{2}}=-2 a_{i i}<0
$$

This completes the proof.

According to the above results, we have a componentwise splitting of the Helmholtz free energy density with the Van der Waals model into two parts:

$$
f_{0}(\mathbf{n})=\left(f_{0}^{\text {ideal }}(\mathbf{n})+f_{0}^{b}(\mathbf{n})\right)+f_{0}^{a}(\mathbf{n}),
$$

where the first part $\left(f_{0}^{\text {ideal }}(\mathbf{n})+f_{0}^{b}(\mathbf{n})\right)$ is convex with $n_{i}$ and $f_{0}^{a}(\mathbf{n})$ is concave with respect to $n_{i}$.

3.2.2. The componentwise convex splitting of $f_{0}$ with the PengRobinson model. For the Peng-Robinson model, the "ideal" and repulsion contributions to the Helmholtz free energy density are the same as the Van der Waals expression, but the major difference is in the attraction term that we consider in this section.

For convenience of analysis, we adopt the following notations. Denote $\tilde{b}$ by $\sum_{j=1}^{M} b_{j} n_{j}, \tilde{a}_{i}$ by $\sum_{j=1}^{M} a_{i j} n_{j}$, and $\tilde{a}$ by $\sum_{j=1}^{M} \sum_{k=1}^{M} a_{j k} n_{k} n_{j}$. By the definitions of $a_{i}$, $b_{i}$, and $n_{i}$, they must be bounded by $\min _{i} a_{i} \leq a_{i} \leq \max _{i} a_{i}, \min _{i} b_{i} \leq b_{i} \leq \max _{i} b_{i}$, and $\min _{i} n_{i} \leq n_{i} \leq \max _{i} n_{i}$, respectively. Here the boundedness of $n_{i}$ is because of the finite amount of each species and the limited volume of the domain. Obviously, $\tilde{b}$ must also be bounded by $\min \tilde{b} \leq \tilde{b} \leq \max \tilde{b}, \tilde{a}_{i}$ by $\min _{i} \tilde{a}_{i} \leq \tilde{a}_{i} \leq \max _{i} \tilde{a}_{i}$, and $\tilde{a}$ by $\min \tilde{a} \leq \tilde{a} \leq \max \tilde{a}$.

Now let

$$
\tilde{f}_{0}(\mathbf{n})=f_{0}^{\text {ideal }}(\mathbf{n})+f_{0}^{b}(\mathbf{n})+f_{0}^{a}(\mathbf{n})+\frac{1}{2} K \widehat{a} n^{2}-\frac{1}{2} K \widehat{a} n^{2},
$$

where $f_{0}^{\text {ideal }}(\mathbf{n})=R T \sum_{i=1}^{M} n_{i}\left(\ln n_{i}-1\right), f_{0}^{b}(\mathbf{n})=-n R T \ln (1-b n), f_{0}^{a}(\mathbf{n})=$ $\frac{\widetilde{a}}{2 \sqrt{2} \tilde{b}} \ln \left(\frac{1+(1-\sqrt{2}) \tilde{b}}{1+(1+\sqrt{2}) \widetilde{b}}\right), \widehat{a}=\sum_{i, j=1}^{M} \sqrt{a_{i} a_{j}} x_{i} x_{j}, a_{i}$ being computed from (A.1) in Appendix A, and $K$ is a constant and will be derived in subsequent sections.

REMARK 3.1. Here the term $\frac{1}{2} K \widehat{a} n^{2}$ is analogous to $-a n^{2}$, which represents the energy density from attraction in the Van der Waals model, but here $k_{i j}=0$ in the mixing rule. As shown, adding this term to the Peng-Robinson model is physicspreserved and does not change the Helmholtz free energy density. More importantly, $\widehat{A}=\left(\widehat{a}_{i j}\right)_{M \times M}=\left(\sqrt{a_{i} a_{j}}\right)_{M \times M}$ is positive semidefinite and $a_{i}=\widehat{a}_{i i}>0$, which plays an essential role in our numerical algorithms.

Rearrange $\tilde{f}_{0}(\mathbf{n})$ by

$$
\tilde{f}_{0}(\mathbf{n})=\left(f_{0}^{\text {ideal }}(\mathbf{n})+f_{0}^{b}(\mathbf{n})+\frac{1}{2} K \widehat{a} n^{2}\right)+\left(f_{0}^{a}(\mathbf{n})-\frac{1}{2} K \widehat{a} n^{2}\right) .
$$

We have the following lemma for the first part of $\tilde{f}_{0}$.

Lemma 3.5. If the constant $K$ is nonnegative, then $f_{0}^{\text {conv }}=f_{0}^{\text {ideal }}(\mathbf{n})+f_{0}^{b}+$ $\frac{1}{2} K \widehat{a} n^{2}$ is componentwise convex with $n_{i}$; otherwise stated, $\frac{\partial^{2} f_{0}^{\text {conv }}}{\partial n_{i}^{2}}>0$.

Copyright $@$ by SIAM. Unauthorized reproduction of this article is prohibited. 
Proof. The proof is straightforward:

$$
\frac{\partial^{2} f_{0}^{\text {conv }}}{\partial n_{i}^{2}}=\frac{\partial^{2} f_{0}^{\text {ideal }}}{\partial n_{i}^{2}}+\frac{\partial^{2} f_{0}^{b}}{\partial n_{i}^{2}}+K \widehat{a}_{i i}
$$

from Lemmas 3.2 and $3.3, \frac{\partial^{2} f_{0}^{\text {ideal }}}{\partial n_{i}^{2}}+\frac{\partial^{2} f_{0}^{b}}{\partial n_{i}^{2}}>0$, and $K \geq 0$, so $\frac{\partial^{2} f_{0}^{\text {conv }}}{\partial n_{i}^{2}}>0$.

For the second part of $\tilde{f}_{0}$ we have the following lemma.

LEMMA 3.6. If we let

$$
\begin{aligned}
K \geq \max \left\{-\frac{b_{i}\left(2 \tilde{a}_{i} \tilde{b}-b_{i} \tilde{a}\right)}{\sqrt{2} a_{i} \tilde{b}^{3}} \ln \left(\frac{1+(1-\sqrt{2}) \tilde{b}}{1+(1+\sqrt{2}) \tilde{b}}\right)\right. \\
\left.-\frac{2 b_{i}\left(2 \tilde{b}^{\tilde{a}}\left(1+2 \tilde{b}-\tilde{b}^{2}\right)-b_{i} \tilde{a}\left(1+3 \tilde{b}-2 \tilde{b}^{2}\right)\right)}{a_{i} \tilde{b}^{2}(1+(1-\sqrt{2}) \tilde{b})^{2}(1+(1+\sqrt{2}) \tilde{b})^{2}}, 0\right\},
\end{aligned}
$$

then $\widetilde{f}_{0}^{a}(\mathbf{n})=f_{0}^{a}(\mathbf{n})-\frac{1}{2} K \widehat{a} n^{2}$ is concave with $n_{i}$, namely, $\frac{\partial^{2} \widetilde{f}_{0}^{a}(\mathbf{n})}{\partial n_{i}^{2}}<0$

Proof. We first calculate $\frac{\partial^{2} f_{0}^{a}}{\partial n_{i}^{2}}$. Recall that $f_{0}^{a}(\mathbf{n})=\frac{\widetilde{a}}{2 \sqrt{2} \tilde{b}} \ln \left(\frac{1+(1-\sqrt{2}) \tilde{b}}{1+(1+\sqrt{2}) \tilde{b}}\right)$, so

$$
\begin{aligned}
\mu_{0, i}^{a}= & \frac{\partial f_{0}^{a}}{\partial n_{i}}=\frac{2 \tilde{b}_{\tilde{a}_{i}}-b_{i} \tilde{a}}{2 \sqrt{2} \tilde{b}^{2}} \ln \left(\frac{1+(1-\sqrt{2}) \tilde{b}}{1+(1+\sqrt{2}) \tilde{b}}\right) \\
& +\frac{\tilde{a}}{2 \sqrt{2} \tilde{b}}\left(\frac{(1-\sqrt{2}) b_{i}}{1+(1-\sqrt{2}) \tilde{b}}-\frac{(1+\sqrt{2}) b_{i}}{1+(1+\sqrt{2}) \tilde{b}}\right)
\end{aligned}
$$

and we have

$$
\begin{aligned}
\frac{\partial^{2} f_{0}^{a}}{\partial n_{i}^{2}}=\frac{\partial \mu_{0, i}^{a}}{\partial n_{i}}= & \frac{a_{i i}}{\sqrt{2} \tilde{b}} \ln \left(\frac{1+(1-\sqrt{2}) \tilde{b}}{1+(1+\sqrt{2}) \tilde{b}}\right)-\frac{b_{i}\left(2 \tilde{a}_{i} \tilde{b}-b_{i} \tilde{a}\right)}{\sqrt{2} \tilde{b}^{3}} \ln \left(\frac{1+(1-\sqrt{2}) \tilde{b}}{1+(1+\sqrt{2}) \tilde{b}}\right) \\
& -\frac{2 b_{i}\left(2 \tilde{b} \tilde{a}_{i}\left(1+2 \tilde{b}-\tilde{b}^{2}\right)-b_{i} \tilde{a}\left(1+3 \tilde{b}-2 \tilde{b}^{2}\right)\right)}{\tilde{b}^{2}(1+(1-\sqrt{2}) \tilde{b})^{2}(1+(1+\sqrt{2}) \tilde{b})^{2}} .
\end{aligned}
$$

Eventually, we get

$$
\begin{aligned}
\frac{\partial^{2} \tilde{f}_{0}^{a}}{\partial n_{i}^{2}} & =\frac{\partial^{2} f_{0}^{a}}{\partial n_{i}^{2}}-K \widehat{a}_{i i} \\
& =\frac{a_{i i}}{\sqrt{2} \tilde{b}} \ln \left(\frac{1+(1-\sqrt{2}) \tilde{b}}{1+(1+\sqrt{2}) \tilde{b}}\right)-\frac{b_{i}\left(2 \tilde{a}_{i} \tilde{b}-b_{i} \tilde{a}\right)}{\sqrt{2} \tilde{b}^{3}} \ln \left(\frac{1+(1-\sqrt{2}) \tilde{b}}{1+(1+\sqrt{2}) \tilde{b}}\right)
\end{aligned}
$$

Copyright (C) by SIAM. Unauthorized reproduction of this article is prohibited. 


$$
\begin{aligned}
& -\frac{2 b_{i}\left(2 \tilde{b}^{\tilde{a}_{i}}\left(1+2 \tilde{b}-\tilde{b}^{2}\right)-b_{i} \tilde{a}\left(1+3 \tilde{b}-2 \tilde{\tilde{b}}^{2}\right)\right)}{\tilde{b}^{2}(1+(1-\sqrt{2}) \tilde{b})^{2}\left(1+(1+\sqrt{2}) \tilde{b}^{2}\right.}-K a_{i} \\
\leq & \frac{a_{i i}}{\sqrt{2} \tilde{b}} \ln \left(\frac{1+(1-\sqrt{2}) \tilde{b}}{1+(1+\sqrt{2}) \tilde{b}}\right)<0 .
\end{aligned}
$$

Here we take the fact that $\widehat{a}_{i i}=a_{i}$ by the definition of $\widehat{a}_{i i}$.

According to the above results, the total Helmholtz free energy density with the Peng-Robinson model can be split into summation of two parts:

$$
\tilde{f}_{0}(\mathbf{n})=\left(f_{0}^{\text {ideal }}(\mathbf{n})+f_{0}^{b}(\mathbf{n})+\frac{1}{2} K \widehat{a} n^{2}\right)+\left(f_{0}^{a}(\mathbf{n})-\frac{1}{2} K \widehat{a} n^{2}\right)
$$

where $K$ satisfies $(3.10)$, the first part $f_{0}^{\text {ideal }}(\mathbf{n})+f_{0}^{b}(\mathbf{n})+\frac{1}{2} K \widehat{a} n^{2}$ is convex, and $f_{0}^{a}(\mathbf{n})-\frac{1}{2} K \widehat{a} n^{2}$ is concave with respect to $n_{i}$.

3.2.3. An energy-stable componentwise convex splitting scheme. For convenience of analysis, let $\mathbf{n}^{k+\frac{i}{M}}$ be a vector of $\left(n_{1}^{k+1}, \ldots, n_{i}^{k+1}, n_{i+1}^{k}, \ldots, n_{M}^{k}\right)$, the $j$ th element of which has the property

$$
n_{j}^{k+\frac{i}{M}}= \begin{cases}n_{j}^{k+1} & \text { if } j \leq i, \\ n_{j}^{k} & \text { if } j>i .\end{cases}
$$

For the system (3.2)-(3.5) with the Van der Waals model we propose an unconditional convex splitting scheme as follows:

$$
\begin{aligned}
\frac{n_{i}^{k+\frac{i}{M}}-n_{i}^{k+\frac{i-1}{M}}}{\delta t}-c_{i i} \Delta n_{i}^{k+\frac{i}{M}}= & \mu_{i}^{k+\frac{i}{M}}-\left(\mu_{0, i}^{\text {ideal }}\left(\mathbf{n}^{k+\frac{i}{M}}\right)+\mu_{0, i}^{b}\left(\mathbf{n}^{k+\frac{i}{M}}\right)\right) \\
& -\mu_{0, i}^{a}\left(\mathbf{n}^{k+\frac{i-1}{M}}\right)+\sum_{j \neq i} c_{i j} \Delta n_{j}^{k+\frac{i}{M}}, i=1, \ldots, M, \\
\int_{\Omega} n_{i}^{k+\frac{i}{M}} d \mathbf{x} & =N_{i}, \quad i=1, \ldots, M, \\
\nabla n_{i}^{k+\frac{i}{M}} \cdot \nu_{\partial \Omega} & =0, \quad \mathbf{x} \in \partial \Omega, \quad i=1, \ldots, M, \\
n_{i}^{0} & =n_{i}^{\text {init }}, \quad i=1, \ldots, M .
\end{aligned}
$$

THEOREM 3.7. The semidiscrete scheme (3.12)-(3.15) is uniformly energy decreasing for each $i$; that is, for any time step size $\delta t>0$ the total Helmholtz free energy satisfies

$$
F\left(\mathbf{n}^{k+\frac{i}{M}}\right) \leq F\left(\mathbf{n}^{k+\frac{i-1}{M}}\right), \quad i=1, \ldots, M .
$$

Proof. A sketch for the proof here is given below. The full proof is described in Appendix C.

Copyright $@$ by SIAM. Unauthorized reproduction of this article is prohibited. 
Taking the inner product of $-\left(n_{i}^{k+\frac{i}{M}}-n_{i}^{k+\frac{i-1}{M}}\right)$ with (3.12), we observe that

$$
\begin{aligned}
& -\frac{1}{\delta t}\left\|n_{i}^{k+\frac{i}{M}}-n_{i}^{k+\frac{i-1}{M}}\right\|^{2} \\
= & \left(c_{i i} \nabla n_{i}^{k+\frac{i}{M}}, \nabla n_{i}^{k+\frac{i}{M}}-\nabla n_{i}^{k+\frac{i-1}{M}}\right)+\left(\sum_{j \neq i} c_{i j} \nabla n_{j}^{k+\frac{i}{M}}, \nabla n_{i}^{k+\frac{i}{M}}-\nabla n_{i}^{k+\frac{i-1}{M}}\right) \\
& +\left(\left.\left(\frac{\partial f_{0}^{\text {ideal }}}{\partial n_{i}}+\frac{\partial f_{0}^{\mathrm{b}}}{\partial n_{i}}\right)\right|_{\mathbf{n}=\mathbf{n}^{k+\frac{i}{M}}}, n_{i}^{k+\frac{i}{M}}-n_{i}^{k+\frac{i-1}{M}}\right) \\
& +\left(\left.\frac{\partial f_{0}^{\mathrm{a}}}{\partial n_{i}}\right|_{\mathbf{n}=\mathbf{n}^{k+\frac{i-1}{M}}}, n_{i}^{k+\frac{i}{M}}-n_{i}^{k+\frac{i-1}{M}}\right) \\
\geq & \frac{1}{2} \sum_{j, l=1}^{M} c_{j l} \nabla n_{j}^{k+\frac{i}{M}} \cdot \nabla n_{l}^{k+\frac{i}{M}}-\frac{1}{2} \sum_{j, l=1}^{M} c_{j l} \nabla n_{j}^{k+\frac{i-1}{M}} \cdot \nabla n_{l}^{k+\frac{i-1}{M}} .
\end{aligned}
$$

$f_{0}^{\text {ideal }}(\mathbf{n})$ is convex with respect to $n_{i}$, and hence

$$
\left(\left.\frac{\partial f_{0}^{\text {ideal }}}{\partial n_{i}}\right|_{\mathbf{n}=\mathbf{n}^{k+\frac{i}{M}}}, n_{i}^{k+\frac{i}{M}}-n_{i}^{k+\frac{i-1}{M}}\right) \geq f_{0}^{\text {ideal }}\left(\mathbf{n}^{k+\frac{i}{M}}\right)-f_{0}^{\text {ideal }}\left(\mathbf{n}^{k+\frac{i-1}{M}}\right) .
$$

Similarly,

$$
\left(\left.\frac{\partial f_{0}^{\mathrm{b}}}{\partial n_{i}}\right|_{\mathbf{n}=\mathbf{n}^{k+\frac{i}{M}}}, n_{i}^{k+\frac{i}{M}}-n_{i}^{k+\frac{i-1}{M}}\right) \geq f_{0}^{\mathrm{b}}\left(\mathbf{n}^{k+\frac{i}{M}}\right)-f_{0}^{\mathrm{b}}\left(\mathbf{n}^{k+\frac{i-1}{M}}\right) .
$$

$f_{0}^{\mathrm{a}}$ is concave with respect to $n_{i}$, so

$$
\left(\left.\frac{\partial f_{0}^{\mathrm{a}}}{\partial n_{i}}\right|_{\mathbf{n}=\mathbf{n}^{k+\frac{i-1}{M}}}, n_{i}^{k+\frac{i}{M}}-n_{i}^{k+\frac{i-1}{M}}\right) \geq f_{0}^{\mathrm{a}}\left(\mathbf{n}^{k+\frac{i}{M}}\right)-f_{0}^{\mathrm{a}}\left(\mathbf{n}^{k+\frac{i-1}{M}}\right) .
$$

Combining the inequalities (3.17)-(3.20), we have

$$
0 \geq f\left(\mathbf{n}^{k+\frac{i}{M}}\right)-f\left(\mathbf{n}^{k+\frac{i-1}{M}}\right) .
$$

Integration yields

$$
F\left(\mathbf{n}^{k+\frac{i}{M}}\right) \leq F\left(\mathbf{n}^{k+\frac{i-1}{M}}\right)
$$

This completes the proof.

Corollary 1. From Theorem 3.7 it is straightforward to find that the semidiscrete scheme (3.12)-(3.15) also satisfies

$$
F\left(\mathbf{n}^{k+1}\right) \leq F\left(\mathbf{n}^{k}\right) .
$$

Proof. From (3.21),

$F\left(\mathbf{n}^{k+1}\right)=F\left(\mathbf{n}^{k+\frac{M}{M}}\right) \leq F\left(\mathbf{n}^{k+\frac{M-1}{M}}\right) \leq \cdots \leq F\left(\mathbf{n}^{k+\frac{1}{M}}\right) \leq F\left(\mathbf{n}^{k+\frac{0}{M}}\right)=F\left(\mathbf{n}^{k}\right)$. 
For the Peng-Robinson model, with the newly defined $\tilde{f}_{0},(3.2)$ is equivalent to

$$
\begin{aligned}
\frac{\partial n_{i}}{\partial t}-\sum_{j=1}^{M} c_{i j} \Delta n_{j}= & \mu_{i}(t)-\left(\mu_{0, i}^{\text {ideal }}(\mathbf{n})+\mu_{0, i}^{b}(\mathbf{n})+K \sum_{j=1}^{M} \widehat{a}_{i j} n_{j}\right) \\
& -\left(\mu_{0, i}^{a}(\mathbf{n})-K \sum_{j=1}^{M} \widehat{a}_{i j} n_{j}\right), \quad \mathbf{x} \in \Omega, \quad i=1, \ldots, M
\end{aligned}
$$

We define $\widehat{a}_{i}$ as the row vector $\left(\widehat{a}_{i j}\right)_{j}$. For the system of (3.3)-(3.5), (3.23) we have a componentwise convex splitting procedure given below:

$$
\begin{aligned}
& \frac{n_{i}^{k+\frac{i}{M}}-n_{i}^{k+\frac{i-1}{M}}}{\delta t}-c_{i i} \Delta n_{i}^{k+\frac{i}{M}} \\
&=\mu_{i}^{k+\frac{i}{M}}-\left(\mu_{0, i}^{\text {ideal }}\left(\mathbf{n}^{k+\frac{i}{M}}\right)+\mu_{0, i}^{b}\left(\mathbf{n}^{k+\frac{i}{M}}\right)+K \widehat{a}_{i} \mathbf{n}^{k+\frac{i}{M}}\right) \\
&-\left(\mu_{0, i}^{a}\left(\mathbf{n}^{k+\frac{i-1}{M}}\right)-K \widehat{a}_{i} \mathbf{n}^{k+\frac{i-1}{M}}\right)+\sum_{j \neq i} c_{i j} \Delta n_{j}^{k+\frac{i}{M}}, \\
& \int_{\Omega} n_{i}^{k+\frac{i}{M}} d \mathbf{x}=N_{i} \\
& \nabla n_{i}^{k+\frac{i}{M}} \cdot \nu_{\partial \Omega}=0, \quad \mathbf{x} \in \partial \Omega, \\
& n_{i}^{0}=n_{i}^{\text {init }}, \quad \mathbf{x} \in \Omega .
\end{aligned}
$$

TheOREM 3.8. The semidiscrete scheme (3.24)-(3.27) is always energy decaying for each $i$, meaning that for any time step size $\delta t>0$ the total Helmholtz free energy satisfies

$$
F\left(\mathbf{n}^{k+\frac{i}{M}}\right) \leq F\left(\mathbf{n}^{k+\frac{i-1}{M}}\right)
$$

Proof. The inner product of $-\left(n_{i}^{k+\frac{i}{M}}-n_{i}^{k+\frac{i-1}{M}}\right)$ with the $i$ th equation of (3.24) yields

$$
\begin{aligned}
& -\frac{1}{\delta t}\left\|n_{i}^{k+\frac{i}{M}}-n_{i}^{k+\frac{i-1}{M}}\right\|^{2} \\
= & -\left(\mu_{i}^{k+\frac{i}{M}}+\sum_{j=1}^{M} c_{i j} \Delta n_{j}^{k+\frac{i}{M}}, n_{i}^{k+\frac{i}{M}}-n_{i}^{k+\frac{i-1}{M}}\right) \\
& +\left(\mu_{0, i}^{\text {ideal }}\left(\mathbf{n}^{k+\frac{i}{M}}\right)+\mu_{0, i}^{\mathrm{b}}\left(\mathbf{n}^{k+\frac{i}{M}}\right)+K \widehat{a}_{i} \mathbf{n}^{k+\frac{i}{M}}, n_{i}^{k+\frac{i}{M}}-n_{i}^{k+\frac{i-1}{M}}\right) \\
& +\left(\mu_{0, i}^{\mathrm{a}}\left(\mathbf{n}^{k+\frac{i-1}{M}}\right)-K \widehat{a}_{i} \mathbf{n}^{k+\frac{i-1}{M}}, n_{i}^{k+\frac{i}{M}}-n_{i}^{k+\frac{i-1}{M}}\right) \\
= & \left(\sum_{j=1}^{M} c_{i j} \nabla n_{j}^{k+\frac{i}{M}}, \nabla n_{i}^{k+\frac{i}{M}}-\nabla n_{i}^{k+\frac{i-1}{M}}\right) \\
& +\left(\left.\left(\frac{\partial f_{0}^{\text {ideal }}}{\partial n_{i}}+\frac{\partial f_{0}^{\mathrm{b}}}{\partial n_{i}}+K \widehat{a}_{i} \mathbf{n}\right)\right|_{\mathbf{n}=\mathbf{n}^{k+\frac{i}{M}}}, n_{i}^{k+\frac{i}{M}}-n_{i}^{k+\frac{i-1}{M}}\right)
\end{aligned}
$$

Copyright $@$ by SIAM. Unauthorized reproduction of this article is prohibited. 


$$
\begin{aligned}
& +\left(\left.\frac{\partial \tilde{f}_{0}^{\mathrm{a}}}{\partial n_{i}}\right|_{\mathbf{n}=\mathbf{n}^{k+\frac{i-1}{M}}}, n_{i}^{k+\frac{i}{M}}-n_{i}^{k+\frac{i-1}{M}}\right) \\
& +\left\langle\sum_{j \neq i} c_{i j} \nabla n_{j}^{k+\frac{i}{M}} \cdot \nu_{\partial \Omega}, n_{i}^{k+\frac{i}{M}}-n_{i}^{k+\frac{i-1}{M}}\right\rangle_{\partial \Omega} \\
& =\left(\sum_{j=1}^{M} c_{i j} \nabla n_{j}^{k+\frac{i}{M}}, \nabla n_{i}^{k+\frac{i}{M}}-\nabla n_{i}^{k+\frac{i-1}{M}}\right) \\
& +\left(\left.\left(\frac{\partial f_{0}^{\text {ideal }}}{\partial n_{i}}+\frac{\partial f_{0}^{\mathrm{b}}}{\partial n_{i}}+K \widehat{a}_{i} \mathbf{n}\right)\right|_{\mathbf{n}=\mathbf{n}^{k+\frac{i}{M}}}, n_{i}^{k+\frac{i}{M}}-n_{i}^{k+\frac{i-1}{M}}\right) \\
& +\left(\left.\left(\frac{\partial f_{0}^{\mathrm{a}}}{\partial n_{i}}-K \widehat{a}_{i} \mathbf{n}\right)\right|_{\mathbf{n}=\mathbf{n}^{k+\frac{i-1}{M}}}, n_{i}^{k+\frac{i}{M}}-n_{i}^{+\frac{i-1}{M}}\right) .
\end{aligned}
$$

Following the same steps from (3.17)-(3.20) with

$$
\left(\left.K \widehat{a}_{i} \mathbf{n}\right|_{\mathbf{n}=\mathbf{n}^{k+\frac{i}{M}}}, n_{i}^{k+\frac{i}{M}}-n_{i}^{k+\frac{i-1}{M}}\right) \geq K \widehat{a}_{i} \mathbf{n}^{k+\frac{i}{M}}-K \widehat{a}_{i} \mathbf{n}^{k+\frac{i-1}{M}}
$$

and

$$
\left(-\left.K \widehat{a}_{i} \mathbf{n}\right|_{\mathbf{n}=\mathbf{n}^{k+\frac{i-1}{M}}}, n_{i}^{k+\frac{i}{M}}-n_{i}^{k+\frac{i-1}{M}}\right) \geq-\left(K \widehat{a}_{i} \mathbf{n}^{k+\frac{i}{M}}-K \widehat{a}_{i} \mathbf{n}^{k+\frac{i-1}{M}}\right)
$$

we finally obtain

$$
\begin{aligned}
0 \geq & f_{0}\left(\mathbf{n}^{k+\frac{i}{M}}\right)+\frac{1}{2} \sum_{j, l=1}^{M} c_{j l} \nabla n_{j}^{k+\frac{i}{M}} \cdot \nabla n_{l}^{k+\frac{i}{M}} \\
& -\left(f_{0}\left(\mathbf{n}^{k+\frac{i-1}{M}}\right)+\frac{1}{2} \sum_{j, l=1}^{M} c_{j l} \nabla n_{j}^{k+\frac{i-1}{M}} \cdot \nabla n_{l}^{k+\frac{i-1}{M}}\right),
\end{aligned}
$$

and integration of the above inequality implies (3.28).

Corollary 2. From Theorem 3.8 it is easy to see that the semidiscrete scheme (3.24)-(3.27) has the property of the total Helmholtz free energy

$$
F\left(\mathbf{n}^{k+1}\right) \leq F\left(\mathbf{n}^{k}\right) .
$$

Proof. From Theorem 3.8,

$$
\begin{aligned}
F\left(\mathbf{n}^{k+1}\right) & =F\left(\mathbf{n}^{k+\frac{M}{M}}\right) \leq F\left(\mathbf{n}^{k+\frac{M-1}{M}}\right) \leq \cdots \leq F\left(\mathbf{n}^{k+\frac{2}{M}}\right) \\
& \leq F\left(\mathbf{n}^{k+\frac{1}{M}}\right) \leq F\left(\mathbf{n}^{k+\frac{0}{M}}\right)=F\left(\mathbf{n}^{k}\right) .
\end{aligned}
$$

This completes the proof.

3.3. Numerical computation of $\boldsymbol{K}$. In this section, we consider how to numerically compute $K$ occurring in the Peng-Robinson model. It is inappropriate to let $K$ be positively infinite since we need to choose a $K$ such that $\frac{\partial^{2} \widetilde{f}_{0}^{a}}{\partial n_{i}^{2}}$ is negative 
and meanwhile the error introduced by this term should be as small as possible, and therefore theoretically we should find the minimal $K$ satisfying the conditions. We recall the definition of $K$ and let

$$
\begin{aligned}
K_{\min }(\mathbf{n})= & \min \left\{K \mid K \geq \max \left\{-\frac{b_{i}\left(2 \tilde{a}_{i} \tilde{b}-b_{i} \tilde{a}\right)}{\sqrt{2} a_{i} \tilde{b}^{3}} \ln \left(\frac{1+(1-\sqrt{2}) \tilde{b}}{1+(1+\sqrt{2}) \tilde{b}}\right)\right.\right. \\
& \left.\left.-\frac{2 b_{i}\left(2 \tilde{b} \tilde{a}_{i}\left(1+2 \tilde{b}-\tilde{b}^{2}\right)-b_{i} \tilde{a}\left(1+3 \tilde{b}-2 \tilde{b}^{2}\right)\right)}{a_{i} \tilde{b}^{2}\left(1+(1-\sqrt{2}) \tilde{b}^{2}\left(1+(1+\sqrt{2}) \tilde{b}^{2}\right.\right.}, 0\right\}\right\} .
\end{aligned}
$$

In the numerical scheme (3.24), $K$ should be computed for each time step $t^{k+1}$ based on $\mathbf{n}^{k+\frac{i-1}{M}}$, meaning that when computing $n_{i}^{k+\frac{i}{M}}$ (i.e., $\left.n_{i}^{k+1}\right), K=K_{\min }\left(\mathbf{n}^{k+\frac{i}{M}}\right)$. This way, energy decaying of the convex splitting for each component $i$ and minimal error owning to the introduced term can be guaranteed. For efficiency, we take advantage of a numerical scheme $K^{k+\frac{i-1}{M}, l}=(1+\epsilon) K_{\min }\left(\mathbf{n}^{k+\frac{i-1}{M}, l}\right)$ to update $K^{k+\frac{i-1}{M}, l}$, where $\epsilon$ is usually a fixed real number taken from interval $(0,1)$. Pseudocode for computing $K_{\min }\left(\mathbf{n}^{k+\frac{i}{M}}\right)$ is described in Algorithm 1 .

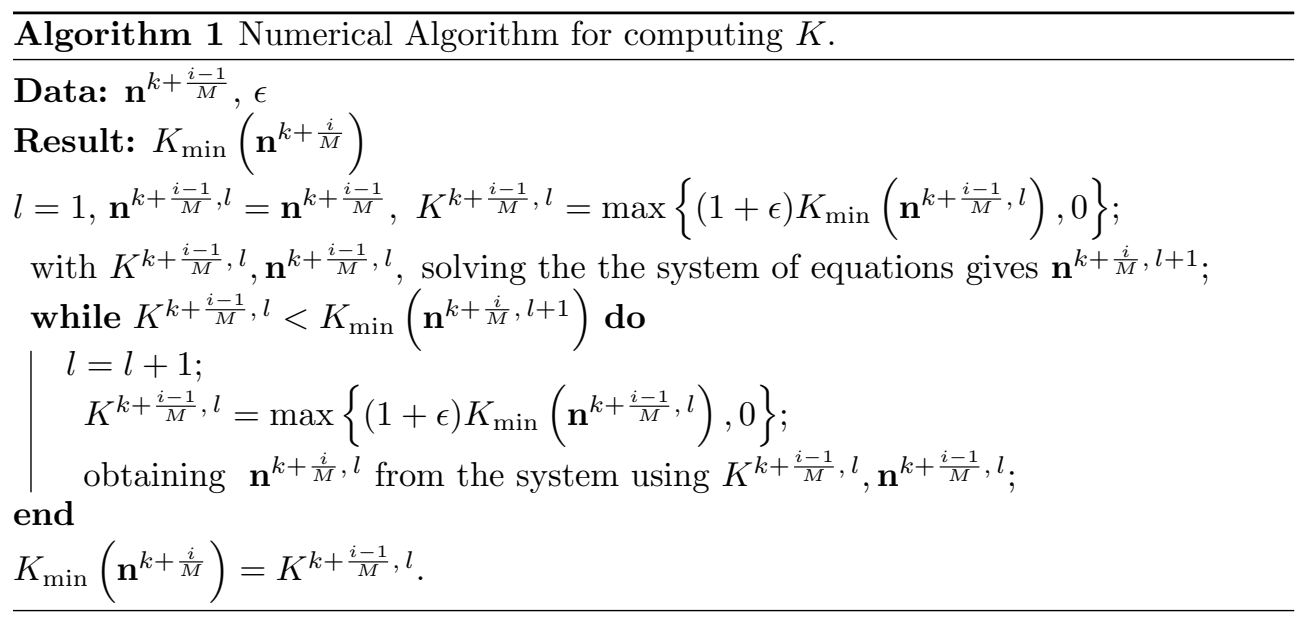

3.4. The mixed finite element approximation for spatial discretization. In the present section, we adopt the RT mixed finite element method to spatially discretize the system (3.2)-(3.5) which has been widely applied for solving elliptic and parabolic partial differential equations, which can guarantee local mass conservation, as we wish here. For simplicity without loss of generality, the domain $\Omega$ is considered as a two-dimensional domain $(d=2)$ divided into triangular meshes. Let $\mathcal{T}_{h}$ be the partition of $\Omega$. Denote $\Gamma_{h}$ by the interior edges of $\mathcal{T}_{h}$. There is a fixed unit normal vector $\nu_{e}$ for each edge $e \in \Gamma_{h}$; it is identical with the outward unit normal vector on 
the boundary of the domain. Define

$$
\begin{aligned}
& \mathbf{V} \equiv H(\Omega ; \operatorname{div}) \equiv\left\{\mathbf{v} \in\left(L^{2}(\Omega)\right)^{d}: \nabla \cdot \mathbf{v} \in L^{2}(\Omega)\right\} \\
& W \equiv L^{2}(\Omega) .
\end{aligned}
$$

Denote by $(\cdot, \cdot)$ and $\langle\cdot, \cdot\rangle_{\Gamma}$ the inner product in $\left(L^{2}(\Omega)\right)^{d}$ or $L^{2}(\Omega)$ and $L^{2}(\Gamma)$, respectively. Since the Van der Waals and the Peng-Robinson models have the same representation in form, for convenience, we will formulate them in a united formula; when the Van der Waals model gets involved, $K \equiv 0$.

Now a weak formulation considered uniformly for the system of (3.3)-(3.5), (3.23) is to search for solution $n_{i} \in W$ and $\mathbf{u}_{i} \in \mathbf{V}$ such that

$$
\begin{aligned}
\left(\frac{\partial n_{i}}{\partial t}, w_{i}\right)= & \left(\mathbf{u}_{i}, \nabla w_{i}\right)-\sum_{E \in \mathcal{T}_{h}}\left\langle\mathbf{u}_{i} \cdot \nu_{\partial E}, w_{i}\right\rangle_{\partial E} \\
& +\left(\mu_{i}(t)-\mu_{0, i}(\mathbf{n}), w_{i}\right) \quad \forall w_{i} \in W, \\
\left(\mathbf{u}_{i}, \mathbf{v}_{i}\right)= & \left(\sum_{j=1}^{M} c_{i j} n_{j}, \nabla \cdot \mathbf{v}_{i}\right) \quad \forall \mathbf{v}_{i} \in \mathbf{V}, \\
\int_{\Omega} n_{i} d \mathbf{x}= & N_{i}, \\
\left(n_{i}, w_{i}\right)= & \left(n_{i}^{\text {init }}, w_{i}\right), \quad t=0 \quad \forall w_{i} \in W
\end{aligned}
$$

where we choose $\mathbf{v}_{i}$ such that $\mathbf{v}_{i} \cdot \nu_{\partial \Omega}=0$ on the domain boundary.

The RT space of $r$ th order $(r \geq 0), R T_{r}$, of the partition $\mathcal{T}_{h}$ is adopted to approximate the subspace $\mathbf{V}_{r}\left(\mathcal{T}_{h}\right) \times W_{r}\left(\mathcal{T}_{h}\right)$ of $\mathbf{V} \times W$. For a two-dimensional domain (i.e., $d=2)$, it is defined as

$$
\begin{aligned}
\mathbf{V}_{r}\left(\mathcal{T}_{h}\right) & =\left\{\mathbf{v} \in H(\Omega ; \operatorname{div}):\left.\mathbf{v}\right|_{\mathrm{E}} \in \mathrm{Q}_{\mathrm{r}+1, \mathrm{r}}(\mathrm{E}) \times \mathrm{Q}_{\mathrm{r}, \mathrm{r}+1}(\mathrm{E}), \mathrm{E} \in \mathcal{T}_{\mathrm{h}}\right\}, \\
W_{r}\left(\mathcal{T}_{h}\right) & =\left\{w \in L^{2}(\Omega):\left.w\right|_{E} \in Q_{r, r+1}(E), E \in \mathcal{T}_{h}\right\},
\end{aligned}
$$

where $Q_{m, n}(E)$ indicates the space of polynomials of degree less than or equal to $m(\mathrm{n})$ in the first (or second) variable restricted to $E . R T_{0}$ is often applied and is utilized in our numerical examples.

We state the mixed finite element approximation to the system of (3.3)-(3.5), (3.23) as follows: to search for $n_{i, h} \in W_{r}\left(\mathcal{T}_{h}\right)$ and $\mathbf{u}_{i, h} \in \mathbf{V}_{r}\left(\mathcal{T}_{h}\right)$ such that

$$
\begin{aligned}
\sum_{E \in \mathcal{T}_{h}}\left(\frac{\partial n_{i, h}}{\partial t}, w_{i, h}\right)_{E}= & \sum_{E \in \mathcal{T}_{h}}\left(\mathbf{u}_{i, h}, \nabla w_{i, h}\right)_{E}-\sum_{E \in \mathcal{T}_{h}}\left\langle\mathbf{u}_{i, h} \cdot \nu_{\partial E}, w_{i, h}\right\rangle_{\partial E} \\
& +\sum_{E \in \mathcal{T}_{h}}\left(\mu_{i}(t)-\mu_{0, i}(\mathbf{n}), w_{i, h}\right)_{E} \quad \forall w_{i, h} \in W_{r}\left(\mathcal{T}_{h}\right), \\
\sum_{E \in \mathcal{T}_{h}}\left(\mathbf{u}_{i, h}, \mathbf{v}_{i, h}\right)_{E}= & \sum_{E \in \mathcal{T}_{h}}\left(\sum_{j=1}^{M} c_{i j} n_{j, h}, \nabla \cdot \mathbf{v}_{i, h}\right)_{E} \quad \forall \mathbf{v}_{i, h} \in \mathbf{V}_{r}\left(\mathcal{T}_{h}\right), \\
\sum_{E \in \mathcal{T}_{h}} \int_{E} n_{i, h} d \mathbf{x}= & N_{i}, \\
\left(n_{i, h}, w_{i, h}\right)_{E} & =\left(n_{i, h}^{\text {init }}, w_{i, h}\right)_{E}, t=0 \quad \forall w_{i, h} \in W_{r}\left(\mathcal{T}_{h}\right),
\end{aligned}
$$

Copyright (c) by SIAM. Unauthorized reproduction of this article is prohibited. 
where we still have to choose $\mathbf{v}_{i, h}$ such that $\mathbf{v}_{i, h} \cdot \nu_{\partial \Omega}=0$ on the domain boundary.

The full discretization of (3.3)-(3.5), (3.23) by combining the componentwise convex splitting semi-implicit method with the mixed finite element approximation is stated as follows: to search for $n_{i, h}^{k+\frac{i}{M}} \in W_{r}\left(\mathcal{T}_{h}\right)$ and $\mathbf{u}_{i, h}^{k+\frac{i}{M}} \in \mathbf{V}_{r}\left(\mathcal{T}_{h}\right)$ such that

$$
\begin{aligned}
& \sum_{E \in \mathcal{T}_{h}}\left(\frac{n_{i, h}^{k+\frac{i}{M}}-n_{i, h}^{k+\frac{i-1}{M}}}{\delta t}, w_{i, h}\right)_{E}=\sum_{E \in \mathcal{T}_{h}}\left(\mathbf{u}_{i, h}^{k+\frac{i}{M}}, \nabla w_{i, h}\right)_{E} \\
& -\sum_{E \in \mathcal{T}_{h}}\left\langle\mathbf{u}_{i, h}^{k+\frac{i}{M}} \cdot \nu_{\partial E}, w_{i, h}\right\rangle_{\partial E} \\
& +\sum_{E \in \mathcal{T}_{h}}\left(\mu_{i}^{k+\frac{i}{M}}-\mu_{0, i}^{\text {ideal }}\left(\mathbf{n}^{k+\frac{i}{M}}\right)-\mu_{0, i}^{\mathrm{b}}\left(\mathbf{n}^{k+\frac{i}{M}}\right)\right. \\
& \left.-K \widehat{a}_{i} \mathbf{n}^{k+\frac{i}{M}}, w_{i, h}\right)_{E} \\
& -\sum_{E \in \mathcal{T}_{h}}\left(\mu_{0, i}^{\mathrm{a}}\left(\mathbf{n}^{k+\frac{i-1}{M}}\right)-K \widehat{a}_{i} \mathbf{n}^{k+\frac{i-1}{M}}, w_{i, h}\right)_{E} \\
& \forall w_{i, h} \in W_{r}\left(\mathcal{T}_{h}\right) \text {, } \\
& \sum_{E \in \mathcal{T}_{h}}\left(\mathbf{u}_{i, h}^{k+\frac{i}{M}}, \mathbf{v}_{i, h}\right)_{E}=\sum_{E \in \mathcal{T}_{h}}\left(\sum_{j=1}^{M} c_{i j} n_{j, h}^{k+\frac{i}{M}}, \nabla \cdot \mathbf{v}_{i, h}\right)_{E} \\
& \forall \mathbf{v}_{i, h} \in \mathbf{V}_{r}\left(\mathcal{T}_{h}\right), \\
& \sum_{E \in \mathcal{T}_{h}} \int_{E} n_{i, h}^{k+\frac{i}{M}} d \mathbf{x}=N_{i} \\
& \left(n_{i, h}^{0}, w_{i, h}\right)=\left(n_{i}^{\text {init }}, w_{i, h}\right) \quad \forall w_{i, h} \in W_{r}\left(\mathcal{T}_{h}\right),
\end{aligned}
$$

where $\mathbf{v}_{i, h}$ satisfies $\mathbf{v}_{i, h} \cdot \nu_{\partial \Omega}=0$ on the domain boundary.

Proposition 1. The fully discretized componentwise convex splitting scheme (3.46)(3.49) is unconditionally stable; in other words, for any time step $\delta t>0$, the following inequality holds:

$$
F_{h}\left(\mathbf{n}_{h}^{k+\frac{i}{M}}\right) \leq F_{h}\left(\mathbf{n}_{h}^{k+\frac{i-1}{M}}\right)
$$

and furthermore,

$$
F_{h}\left(\mathbf{n}_{h}^{k+1}\right) \leq F_{h}\left(\mathbf{n}_{h}^{k}\right)
$$

where $F_{h}\left(\mathbf{n}_{h}^{k+\frac{i}{M}}\right)$ is defined as

$$
F_{h}\left(\mathbf{n}_{h}^{k+\frac{i}{M}}\right)=\sum_{E \in \mathcal{T}_{h}} \int_{E}\left(f_{0}\left(\mathbf{n}_{h}^{k+\frac{i}{M}}\right)+\frac{1}{2} \sum_{j, l=1}^{M} c_{j l} \nabla_{h} n_{j, h}^{k+\frac{i}{M}} \cdot \nabla_{h} n_{l, h}^{k+\frac{i}{M}}\right) d \mathbf{x}
$$

and $F_{h}\left(\mathbf{n}_{h}^{k+1}\right)$ as

$$
F_{h}\left(\mathbf{n}_{h}^{k+1}\right)=\sum_{E \in \mathcal{T}_{h}} \int_{E}\left(f_{0}\left(\mathbf{n}_{h}^{k+1}\right)+\frac{1}{2} \sum_{j, l=1}^{M} c_{j l} \nabla_{h} n_{j, h}^{k+1} \cdot \nabla_{h} n_{l, h}^{k+1}\right) d \mathbf{x} .
$$

Copyright $@$ by SIAM. Unauthorized reproduction of this article is prohibited. 
Proof. Following the same steps of proof as in Theorem 3.8 with the fully discretized system (3.46)-(3.49) and the discrete energy (3.52) yields

$$
F_{h}\left(\mathbf{n}_{h}^{k+\frac{i}{M}}\right)-F_{h}\left(\mathbf{n}_{h}^{k+\frac{i-1}{M}}\right) \leq-\frac{1}{\delta t} \sum_{E \in \mathcal{T}_{h}}\left\|n_{i, h}^{k+\frac{i}{M}}-n_{i, h}^{k+\frac{i-1}{M}}\right\|^{2} \leq 0
$$

and the above inequality gives

$$
\begin{aligned}
F_{h}\left(\mathbf{n}_{h}^{k+1}\right) & =F_{h}\left(\mathbf{n}_{h}^{k+\frac{M}{M}}\right) \leq F_{h}\left(\mathbf{n}_{h}^{k+\frac{M-1}{M}}\right) \leq \cdots \leq F_{h}\left(\mathbf{n}_{h}^{k+\frac{1}{M}}\right) \\
& \leq F_{h}\left(\mathbf{n}_{h}^{k+\frac{0}{M}}\right)=F_{h}\left(\mathbf{n}_{h}^{k}\right) .
\end{aligned}
$$

This completes the proof.

4. Numerical examples. In this section, a two-phase (liquid and gas phases) binary component fluid mixture is simulated to verify the proposed numerical schemes. The mixture consists of methane $\left(\mathrm{CH}_{4}\right)$ and n-decane $\left(n \mathrm{C}_{10} \mathrm{H}_{22}\right)$. The temperature of the whole domain stays constant at $450 \mathrm{~K}$ during the simulation. Some relevant property parameters for the species are listed in Table 1 . The binary interaction parameters are $k_{11}=k_{22}=0$ and $k_{12}=k_{21}=0.052134$. The simulation is carried out on a disk domain with diameter $L=10^{-8}$ meters based on the model of (3.2)(3.5). The domain is divided into 2990 triangles with 1539 nodes; see Figure 1a. The mesh size is about $0.25 \times 10^{-9}$ meters. The boundary condition is exactly set as the zero Neumann condition (3.4) on the whole boundary (i.e., the most outer circle of the disk), meaning that there is no mass exchange between the system and environment. The initial condition is to impose liquid molar densities of both components in the square, and a gas mixture is filled in the rest of the domain; see Figures 1a and 1b.

TABLE 1

Property parameters of methane and n-decane (data from Table 3.1 on page 141 of [11]).

\begin{tabular}{lcccc}
\hline Component name & Symbol & $T_{c}(\mathrm{~K})$ & $P_{c}(\mathrm{MPa})$ & $T_{b}(\mathrm{~K})$ \\
\hline methane & $\mathrm{CH}_{4}$ & 190.58 & 4.604 & 111.63 \\
n-decane & $n \mathrm{C}_{10} \mathrm{H}_{22}$ & 617.7 & 2.099 & 447.3 \\
\hline
\end{tabular}

The time step size is uniformly taken as a relatively large one $\left(\delta t=10^{10}\right.$ seconds) since the numerical scheme is unconditionally stable and we want the simulation time to go infinite approaching the steady state solution. Newton's method is adopted as a nonlinear solver with three iterates or with a relative error $r_{e}=10^{-8}$, which is sufficient for our case. In our simulation, homogeneous contribution of free energy is computed based on the Peng-Robinson model.

\subsection{Simulation results.}

- Molar densities and chemical potential distribution. Figures 1a-1b, 2a-2c, and $3 \mathrm{a}-3 \mathrm{c}$ show the evolution of methane's and n-decane's molar density distributions, respectively. Initially, the molar density of both components (see Figures 1a and 1b) jump from a liquid to a gas phase area, which results in an extremely large (infinite in theory) gradient term of the total Helmholtz free energy of the whole fluid system. After five time steps shown in Figures 2a and 3a, there exists a clear gas-liquid interface (in green online only) with a certain thickness and the corner of the square becomes a little rounder than that at the initial condition for both components. Simulation after 10 time 


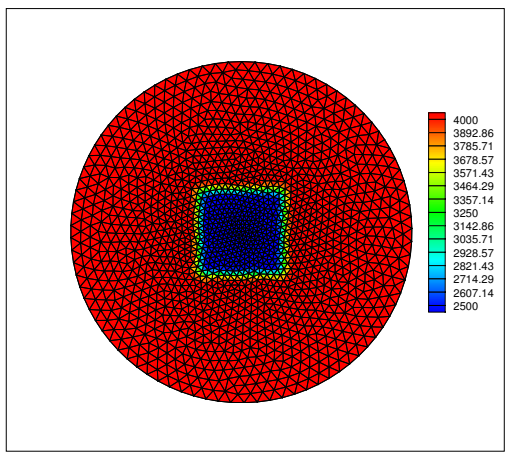

(a)

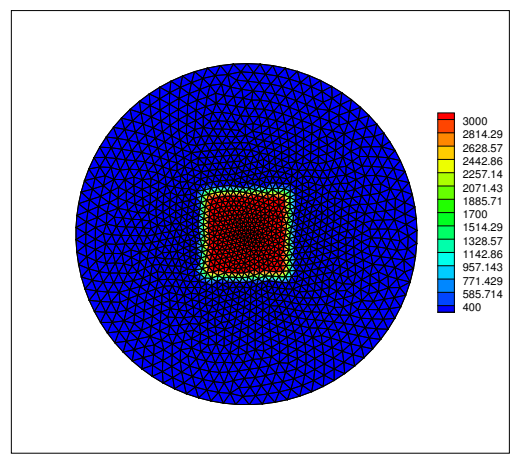

(b)

FIG. 1. Initial condition: (a) molar density of methane; (b) molar density of n-decane.

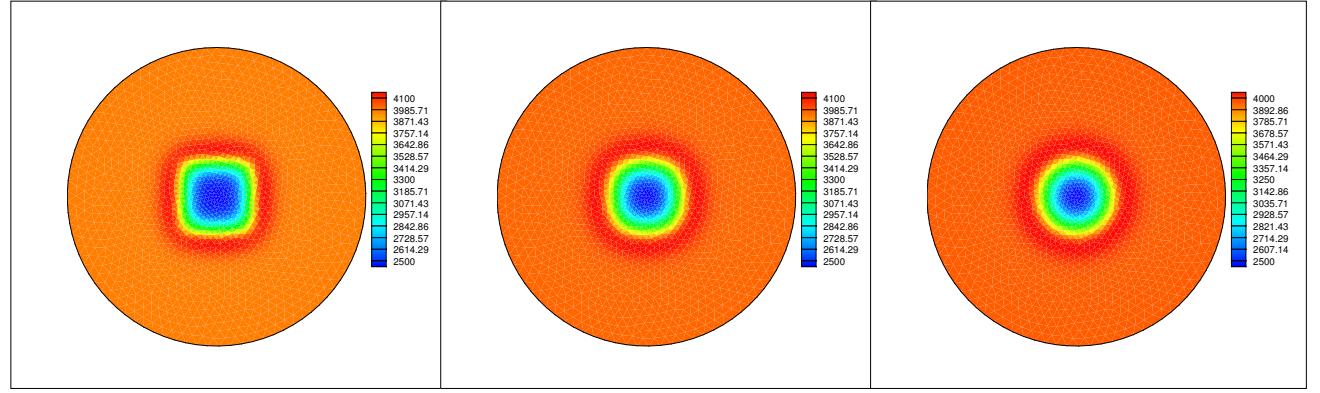

(a) (b) (c)

FIG. 2. Molar density distribution of methane: (a) after five time steps; (b) after 10 time steps; (c) after 20 time steps.

steps results in the corner of the interface becoming much rounder; see Figures $2 \mathrm{~b}$ and $3 \mathrm{~b}$. Finally, after 20 time steps the solution converges and the interface becomes continuous and seems to be a perfectly small circle with very small thickness for methane and n-decane, respectively; see Figures 2c and 3c.

Correspondingly, the homogeneous contribution of chemical potential $\mu_{0}$ of both components changes with the molar densities. Figures $4 \mathrm{a}-4 \mathrm{c}$ and Figures $5 \mathrm{a}-5 \mathrm{c}$ illustrate the evolution of $\mu_{0}$ of methane and n-decane, respectively. $\mu_{0}$ is uniformly the same in the liquid and gas bulk phases. However, it varies sharply across the interface for both species, as expected.

- Decaying of total Helmholtz free energy. It is obviously shown in Figure 6a that the total Helmholtz free energy reduces componentwise at each time step. From the figures we find out that at the first two steps, it plunges quickly; after that, though still decaying (see Figure $6 \mathrm{~b}$ ), the rate of decrease becomes slow and is numerically approaching an equilibrium state that is the solution that we expect. This fast convergence results from the large time step size.

- Values of $K$. In the simulation, we dynamically calculate $K$ as required using Algorithm 1; all $K$ 's during the simulation are zeros, which means that the attraction term of $f_{0}$ is concave with both $\mathrm{CH}_{4}$ and $n \mathrm{C}_{10} \mathrm{H}_{22}$ and the 


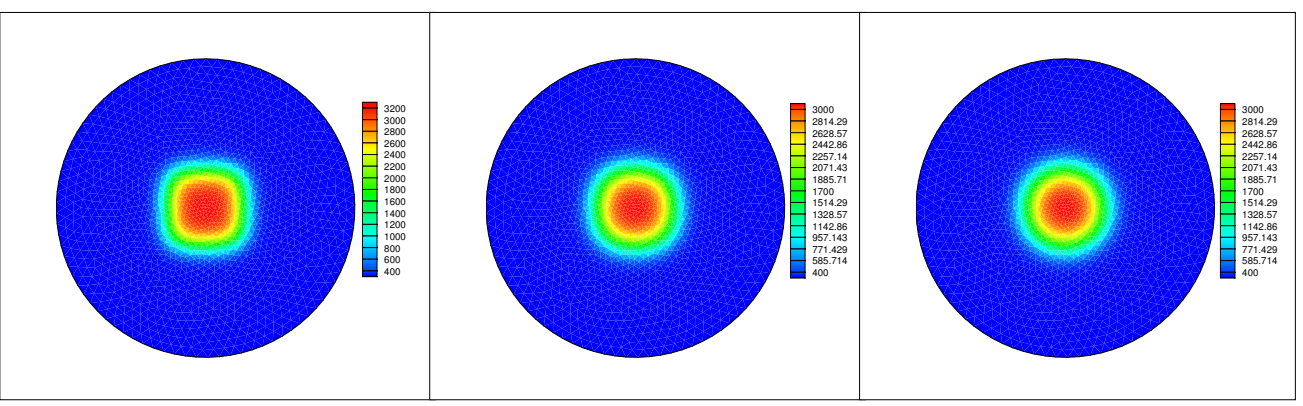

(a)

(b)

(c)

FIG. 3. Molar density distribution of n-decane: (a) after five time steps; (b) after 10 time steps; (c) after 20 time steps.

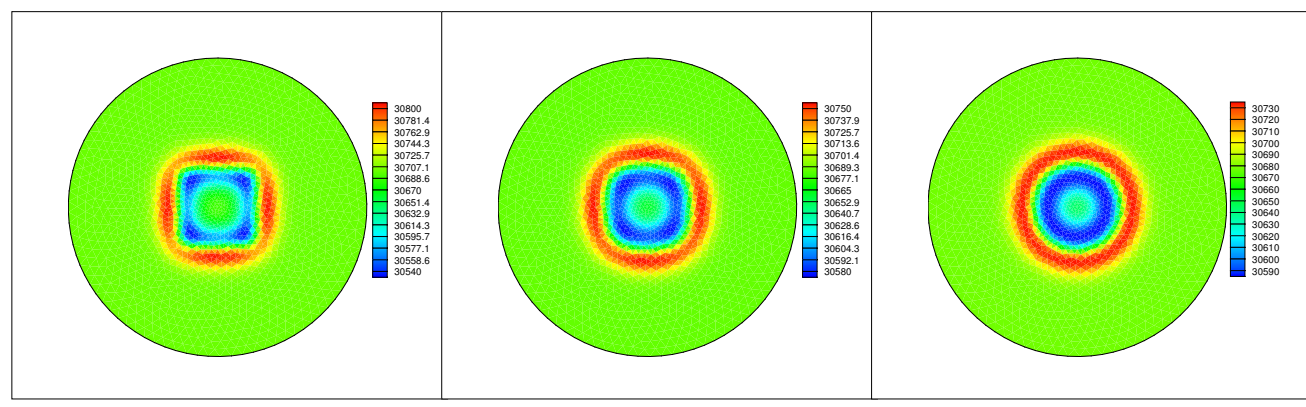

(a)

(b)

(c)

FIG. 4. Chemical potential distribution of methane: (a) after five time steps; (b) after 10 time steps; (c) after 20 time steps.

correction term is not required. In fact, the Peng-Robinson model works well with common-encountered hydrocarbon species in petroleum reservoir, and consequently for most of the hydrocarbon mixtures, the adjustment term is not expected to be used.

4.2. The effect of time step size on convergence. We first compute the results with time step size $\Delta t=10^{6}$ and fine mesh (2990 elements and 1539 nodes and mesh size $\approx 0.25 \times 10^{-9}$ meters) as the approximate exact solution. Furthermore, we obtain the results with two different time step sizes $\Delta t_{1}=5 \times 10^{7}$ and $\Delta t_{2}=5 \times 10^{8}$ on the mesh of 1060 elements and 558 nodes and mesh size $\approx 0.4 \times 10^{-9}$ meters. The energy differences of these two time step sizes with the approximate exact solution are computed, i.e.,

$$
D F_{i}(t)=F_{i}(t)-F_{r}(t), \quad i=1,2,
$$

where $F_{r}(t)$ is the approximate exact solution, and $F_{i}(t)$ is the free energy computed with $\Delta t_{i}$. Following that we calculate the relative error of energy defined as

$$
\operatorname{err}_{i}(t)=\frac{\left|D F_{i}(t)\right|}{F_{r}(t)}, \quad i=1,2 .
$$




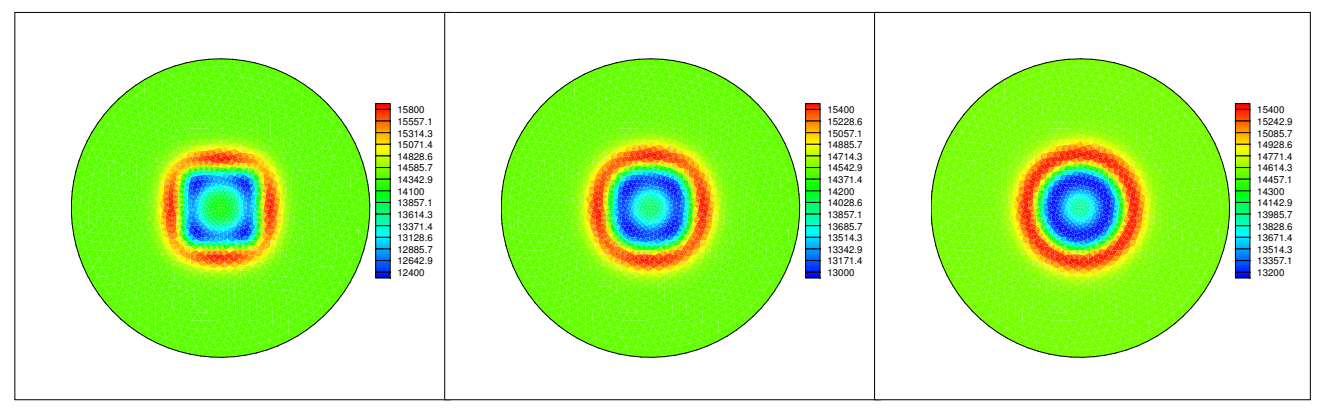

(a)

(b)

(c)

FIG. 5. Chemical potential distribution of n-decane: (a) after five time steps; (b) after 10 time steps; (c) after 20 time steps.

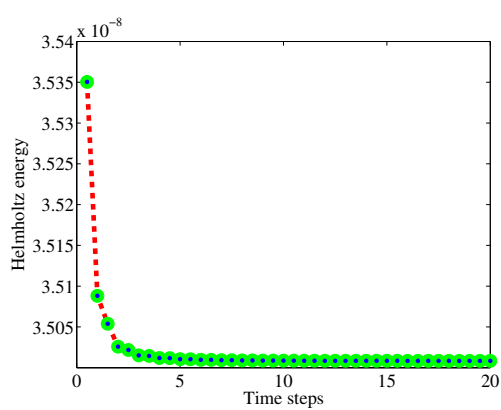

(a)

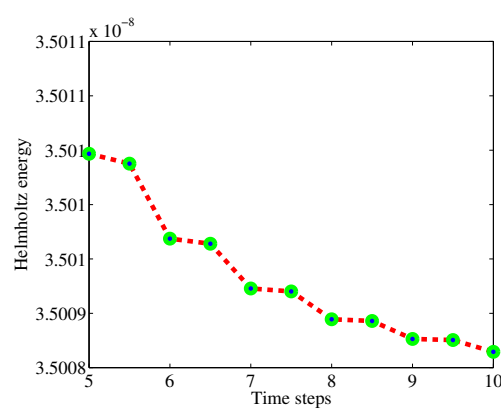

(b)

Fig. 6. Convergence of total Helmholtz free energy: (a) the componentwise total Helmholtz free energy during the whole simulation; (b) zoom in of time steps 5-10.

The result is shown in Figure 7. From this figure, we can say that (1) solutions with both larger time step sizes are convergent to the approximate exact solution; (2) using small time step size the free energy is decreasing more in the same time interval than larger one; however, the one with larger time step size takes many fewer time steps to get nearly the same relative error, which promotes the computational efficiency.

4.3. Comparison of surface tension between laboratory data and computed results. One application of the diffuse interface model is to predict the interface tension. In this section, we calculate the surface tension for the binary mixture we simulated. All of simulations below are carried out on the same domain and the same mesh as in section 4.1 and time step size $\Delta t=10^{10}$. Here the surface tension is computed through the formula $\sigma=\frac{F(\mathbf{n})-F_{0}\left(\mathbf{n}^{\text {init }}\right)}{A}$ provided in [24], where the interface tension is assumed to be constant in the interface. Assume the volume of the droplet is constant from the beginning of simulation to convergence and the liquid droplet is a perfectly round disk when the simulation converges. We compute the interface tension with temperature $280^{\circ} \mathrm{F}$ and pressure from 2000 to 3000 psia every 200 psia; see Figure 8a. And it is also computed at constant pressure 2800 psia and temperatures at $100^{\circ} \mathrm{F}, 200^{\circ} \mathrm{F}$, and $280^{\circ} \mathrm{F}$, respectively; the result is shown in Figure $8 \mathrm{~b}$. The simulated results are compared with the laboratory data from Table 3 of [1] showing 


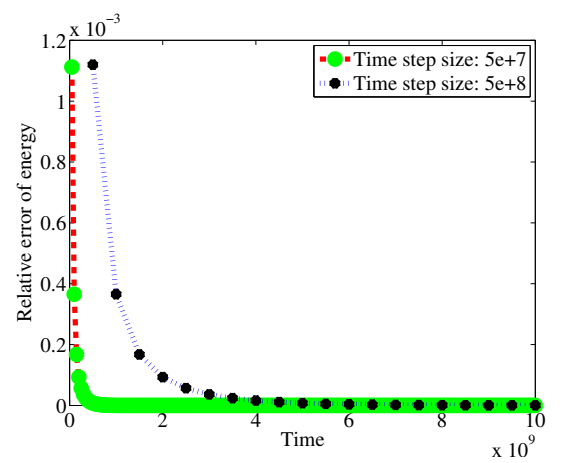

FIG. 7. Relative error of free energy.

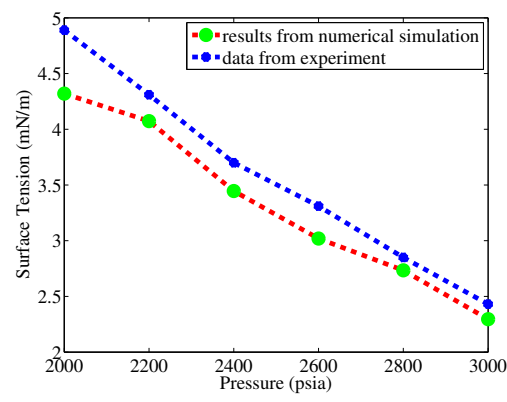

(a)

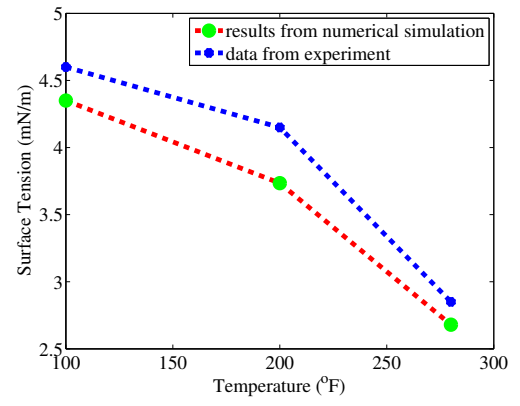

(b)

FIG. 8. Comparison of surface tension between simulations and experimental data: (a) at temperature $T=280^{\circ} \mathrm{F}$; (b) at pressure $P_{0}=2800$ psia.

that the computed results fit well with the measured data to some extent in range of model, measurement, and machine errors.

5. Concluding remarks. We have studied two-phase fluids consisting of multiple components at equilibrium state in a closed system with constant temperature, which are modeled by a gradient theory coupled with the Van der Waals and the PengRobinson EoS. The Van der Waals expression can be elegantly split into summation of convex and concave parts, one of which is borrowed to treat the Peng-Robinson counterpart for the purpose of designing an efficient and robust numerical algorithm. The Peng-Robinson model is more realistic in hydrocarbon mixture applications. The model problem is derived according to the first principle of thermodynamics, which leads to minimizing Helmholtz free energy. With variational calculus and Lagrange multipliers, the minimization problem is equivalently transformed into a system of second-order elliptic partial differential equations of molar density describing the balance of the chemical potential for each species. These equations have strongly nonlinear source terms introduced from the EoS.

The second-order elliptic partial differential equations are converted into a timedependent version by adding a time-derivative term whose solution is sufficiently approached to the original equations when the simulation time goes to infinity. Physicspreserving properties of numerical schemes are always essential for our problem. To 
that end, a componentwise convex splitting semi-implicit scheme is proposed to solve the transient partial differential equations; it discretizes time derivative in a sequential way which is analogous with Gauss-Seidel iteration on time variable but utilizing convex splitting. In addition to its efficiency, the scheme avoids computing the Hessian matrices which have trouble determining its indefiniteness.

More importantly, this strategy guarantees the energy dissipation for the convex splitting step of each component. This method performs perfectly well with the Van der Waals expression of Helmholtz free energy. When the Peng-Robinson model is applied, the attraction term that physically expects to be concave is not easy to judge mathematically. A correction term is plugged into it to take advantage of the componentwise convex splitting strategy. This term formally resembles the concave term in the Van der Waals model and hence is physically consistent. A constant in this correction term is introduced and is numerically updated in each time step for reducing the error and improving the convergence rates.

Numerical examples are tested with real hydrocarbon species to test the theoretical results and efficiency of our proposed algorithm. The numerical results show that all $K$ 's are zeros during the simulation, implying that the correction terms are not needed. In fact, our splitting scheme for the Peng-Robinson model works well with the common-encountered subsurface hydrocarbon mixtures in petroleum engineering. The surface tension is calculated and compared with the laboratory data, which fits well in range of model and measurement errors.

Our future work will include (1) considering three-dimensional problems with gravity; (2) replacing the current EoS to investigate types of fluid mixtures other than hydrocarbon ones; (3) establishing energy decaying matrix splitting schemes for the Van der Waals and Peng-Robinson EoS models.

Appendix A. Parameters of the Van der Waals EoS. For pure-component fluids, $a$ and $b$ for the Van der Waals EoS are derived from the critical state of the species:

$$
a=\frac{27 R^{2} T_{c}^{2}}{64 P_{c}}, \quad b=\frac{R T_{c}}{8 P_{c}},
$$

where $T_{c}$ and $P_{c}$ are the critical temperature and critical pressure, respectively, and for most species they can be attained in engineering applications.

For mixtures, these two parameters are given by the mixing rules associated with the pure species in the fluids:

$$
a=\sum_{i=1}^{M} \sum_{j=1}^{M} x_{i} x_{j}\left(a_{i} a_{j}\right)^{\frac{1}{2}}\left(1-k_{i j}\right), \quad b=\sum_{i=1}^{M} x_{i} b_{i},
$$

where $a_{i}$ and $b_{i}$ stand for the pure-component Van der Waals parameters and $x_{i}$ is the mole ratio of species $i$ defined by $x_{i}=\frac{n_{i}}{n} . k_{i j}$ is the binary interaction coefficient of the Van der Waals EoS; it is given by experimental correlation and is frequently assumed to be constant for a given binary.

According to the thermodynamics theory, the pressure of homogeneous fluids, $p_{0}$, can be computed through the corresponding Helmholtz free energy density $f_{0}(\mathbf{n})$ :

$$
p_{0}=p_{0}(\mathbf{n}, T)=\sum_{i=1}^{M} n_{i}\left(\frac{\partial f_{0}}{\partial n_{i}}\right)-f_{0}=\sum_{i=1}^{M} n_{i} \mu_{0, i}-f_{0} .
$$


Plugging $f_{0}$ and $\mu_{0, i}$ in terms of the Van der Waals expression into (A.2) leads to

$$
p_{0}=\frac{n R T}{1-b n}-a n^{2} .
$$

Appendix B. Parameters of the Peng-Robinson EoS. For pure-component fluid systems, the parameters $a=a(T)$ and $b$ are given by

$$
a=a(T)=0.45724 \frac{R^{2} T_{c}^{2}}{P_{c}}\left(1+m\left(1-\sqrt{T_{r}}\right)\right)^{2}, \quad b=0.07780 \frac{R T_{c}}{P_{c}},
$$

where $T_{r}$ is the reduced temperature defined as $\frac{T}{T_{c}}$ and all the other parameters but $m$ have the same meaning as in the previous section. The parameter $m$ is a fitting formula of the acentric factor $\omega$ of the substance

$$
\begin{aligned}
& m=0.37464+1.5422 \omega-0.26992 \omega^{2}, \quad \omega \leq 0.49 \\
& m=0.379462+1.485030 \omega-0.164423 \omega^{2}+0.016666 \omega^{3}, \quad \omega>0.49 .
\end{aligned}
$$

The acentric factor can be calculated by the critical pressure $P_{c}$, the critical temperature $T_{c}$, and the normal boiling point temperature $T_{b}$ in the following formula:

$$
\omega=\frac{3}{7}\left(\frac{\log _{10}\left(\frac{P_{c}}{1 \mathrm{~atm}}\right)}{\frac{T_{c}}{T_{b}}-1}\right)-1 .
$$

For mixtures, $a=a(T)$ and $b$ are computed by the same mixing rule of (A.1).

Replacing $f_{0}$ and $\mu_{0, i}$ in (A.2) by those of the Peng-Robinson expression, we have the following Peng-Robinson EoS:

$$
p_{0}=\frac{n R T}{1-b n}-\frac{a(T) n^{2}}{1+2 b n-b^{2} n^{2}} .
$$

\section{Appendix C. Complete proof of Theorem 3.7.}

Proof. Taking the inner product of $-\left(n_{i}^{k+\frac{i}{M}}-n_{i}^{k+\frac{i-1}{M}}\right)$ with (3.12), we observe that

$$
\begin{aligned}
& -\frac{1}{\delta t}\left\|n_{i}^{k+\frac{i}{M}}-n_{i}^{k+\frac{i-1}{M}}\right\|^{2} \\
& =-\left(\sum_{j=1}^{M} c_{i j} \Delta n_{j}^{k+\frac{i}{M}}, n_{i}^{k+\frac{i}{M}}-n_{i}^{k+\frac{i-1}{M}}\right)-\left(\mu_{i}^{k+\frac{i}{M}}, n_{i}^{k+\frac{i}{M}}-n_{i}^{k+\frac{i-1}{M}}\right) \\
& +\left(\mu_{0, i}^{\text {ideal }}\left(\mathbf{n}^{k+\frac{i}{M}}\right)+\mu_{0, i}^{\mathrm{b}}\left(\mathbf{n}^{k+\frac{i}{M}}\right), n_{i}^{k+\frac{i}{M}}-n_{i}^{k+\frac{i-1}{M}}\right)+\left(\mu_{0, i}^{\mathrm{a}}\left(\mathbf{n}^{k+\frac{i-1}{M}}\right), n_{i}^{k+\frac{i}{M}}-n_{i}^{k+\frac{i-1}{M}}\right) \\
& =\left(\sum_{j=1}^{M} c_{i j} \nabla n_{j}^{k+\frac{i}{M}}, \nabla n_{i}^{k+\frac{i}{M}}-\nabla n_{i}^{k+\frac{i-1}{M}}\right)+\left(\left.\left(\frac{\partial f_{0}^{\text {ideal }}}{\partial n_{i}}+\frac{\partial f_{0}^{\mathrm{b}}}{\partial n_{i}}\right)\right|_{\mathbf{n}=\mathbf{n}^{k+\frac{i}{M}}}, n_{i}^{k+\frac{i}{M}}-n_{i}^{k+\frac{i-1}{M}}\right) \\
& +\left(\left.\frac{\partial f_{0}^{\mathrm{a}}}{\partial n_{i}}\right|_{\mathbf{n}=\mathbf{n}^{k+\frac{i-1}{M}}}, n_{i}^{k+\frac{i}{M}}-n_{i}^{k+\frac{i-1}{M}}\right)+\left\langle\sum_{j=1}^{M} c_{i j} \nabla n_{j}^{k+\frac{i}{M}} \cdot \nu_{\partial \Omega}, n_{i}^{k+\frac{i}{M}}-n_{i}^{k+\frac{i-1}{M}}\right\rangle_{\partial \Omega} \\
& =\left(\sum_{j=1}^{M} c_{i j} \nabla n_{j}^{k+\frac{i}{M}}, \nabla n_{i}^{k+\frac{i}{M}}-\nabla n_{i}^{k+\frac{i-1}{M}}\right)
\end{aligned}
$$




$$
\begin{aligned}
& +\left(\left.\left(\frac{\partial f_{0}^{\text {ideal }}}{\partial n_{i}}+\frac{\partial f_{0}^{\mathrm{b}}}{\partial n_{i}}\right)\right|_{\mathbf{n}=\mathbf{n}^{k+\frac{i}{M}}}, n_{i}^{k+\frac{i}{M}}-n_{i}^{k+\frac{i-1}{M}}\right)+\left(\left.\frac{\partial f_{0}^{\mathrm{a}}}{\partial n_{i}}\right|_{\mathbf{n}=\mathbf{n}^{k+\frac{i-1}{M}}}, n_{i}^{k+\frac{i}{M}}-n_{i}^{k+\frac{i-1}{M}}\right) \\
& =\left(c_{i i} \nabla n_{i}^{k+\frac{i}{M}}, \nabla n_{i}^{k+\frac{i}{M}}-\nabla n_{i}^{k+\frac{i-1}{M}}\right)+\left(\sum_{j \neq i} c_{i j} \nabla n_{j}^{k+\frac{i}{M}}, \nabla n_{i}^{k+\frac{i}{M}}-\nabla n_{i}^{k+\frac{i-1}{M}}\right) \\
& +\left(\left.\left(\frac{\partial f_{0}^{\text {ideal }}}{\partial n_{i}}+\frac{\partial f_{0}^{\mathrm{b}}}{\partial n_{i}}\right)\right|_{\mathbf{n}=\mathbf{n}^{k+\frac{i}{M}}}, n_{i}^{k+\frac{i}{M}}-n_{i}^{k+\frac{i-1}{M}}\right)+\left(\left.\frac{\partial f_{0}^{\mathrm{a}}}{\partial n_{i}}\right|_{\mathbf{n}=\mathbf{n}^{k+\frac{i-1}{M}}}, n_{i}^{k+\frac{i}{M}}-n_{i}^{k+\frac{i-1}{M}}\right) \\
& \geq \frac{c_{i i}}{2}\left(\left\|\nabla n_{i}^{k+\frac{i}{M}}\right\|^{2}-\left\|\nabla n_{i}^{k+\frac{i-1}{M}}\right\|^{2}\right)+\left(\sum_{j \neq i} c_{i j} \nabla n_{j}^{k+\frac{i}{M}}, \nabla n_{i}^{k+\frac{i}{M}}-\nabla n_{i}^{k+\frac{i-1}{M}}\right) \\
& +\left(\left.\left(\frac{\partial f_{0}^{\text {ideal }}}{\partial n_{i}}+\frac{\partial f_{0}^{\mathrm{b}}}{\partial n_{i}}\right)\right|_{\mathbf{n}=\mathbf{n}^{k+\frac{i}{M}}}, n_{i}^{k+\frac{i}{M}}-n_{i}^{k+\frac{i-1}{M}}\right)+\left(\left.\frac{\partial f_{0}^{\mathrm{a}}}{\partial n_{i}}\right|_{\mathbf{n}=\mathbf{n}^{k+\frac{i-1}{M}}}, n_{i}^{k+\frac{i}{M}}-n_{i}^{k+\frac{i-1}{M}}\right),
\end{aligned}
$$

where

$$
\begin{aligned}
& \frac{c_{i i}}{2}\left(\left\|\nabla n_{i}^{k+\frac{i}{M}}\right\|^{2}-\left\|\nabla n_{i}^{k+\frac{i-1}{M}}\right\|^{2}\right)+\left(\sum_{j \neq i} c_{i j} \nabla n_{j}^{k+\frac{i}{M}}, \nabla n_{i}^{k+\frac{i}{M}}-\nabla n_{i}^{k+\frac{i-1}{M}}\right) \\
= & \frac{c_{i i}}{2}\left\|\nabla n_{i}^{k+\frac{i}{M}}\right\|^{2}+\left(\sum_{j \neq i} c_{i j} \nabla n_{j}^{k+\frac{i}{M}}, \nabla n_{i}^{k+\frac{i}{M}}\right)+\frac{1}{2} \sum_{j, l \neq i-1} c_{j l} \nabla n_{j}^{k+\frac{i}{M}} \cdot \nabla n_{l}^{k+\frac{i}{M}} \\
& -\left(\frac{c_{i i}}{2}\left\|\nabla n_{i}^{k+\frac{i-1}{M}}\right\|^{2}+\left(\sum_{j \neq i} c_{i j} \nabla n_{j}^{k+\frac{i}{M}}, \nabla n_{i}^{k+\frac{i-1}{M}}\right)+\frac{1}{2} \sum_{j, l \neq i-1} c_{j l} \nabla n_{j}^{k+\frac{i}{M}} \cdot \nabla n_{l}^{k+\frac{i}{M}}\right) \\
= & \frac{c_{i i}}{2} \nabla n_{i}^{k+\frac{i}{M}} \cdot \nabla n_{i}^{k+\frac{i}{M}}+\frac{1}{2}\left(\sum_{j \neq i} c_{i j} \nabla n_{j}^{k+\frac{i}{M}}+\sum_{l \neq i} c_{l i} \nabla n_{l}^{k+\frac{i}{M}}\right) \cdot \nabla n_{i}^{k+\frac{i}{M}} \\
& -\left(\frac{c_{i i}}{2} \nabla n_{i}^{k+\frac{i-1}{M}} \cdot \nabla n_{i}^{k+\frac{i-1}{M}}+\frac{1}{2}\left(\sum_{j \neq i} c_{i j} \nabla n_{j}^{k+\frac{i}{M}}+\sum_{l \neq i} c_{l i} \nabla n_{l}^{k+\frac{i}{M}}\right) \cdot \nabla n_{i}^{k+\frac{i-1}{M}}\right) \\
& +\frac{1}{2} \sum_{j, l \neq i-1} c_{j l} \nabla n_{j}^{k+\frac{i-1}{M}} \cdot \nabla n_{l}^{k+\frac{i-1}{M}}-\frac{1}{2} \sum_{j, l \neq i-1} c_{j l} \nabla n_{j}^{k+\frac{i-1}{M}} \cdot \nabla n_{l}^{k+\frac{i-1}{M}} \\
= & \frac{1}{2} \sum_{j, l=1}^{M} c_{j l} \nabla n_{j}^{k+\frac{i}{M}} \cdot \nabla n_{l}^{k+\frac{i}{M}}-\frac{1}{2} \sum_{j, l=1}^{M} c_{j l} \nabla n_{j}^{k+\frac{i-1}{M}} \cdot \nabla n_{l}^{k+\frac{i-1}{M}} .
\end{aligned}
$$

$f_{0}^{\text {ideal }}(\mathbf{n})$ is convex with respect to $n_{i}$, and hence

$$
\left(\left.\frac{\partial f_{0}^{\text {ideal }}}{\partial n_{i}}\right|_{\mathbf{n}=\mathbf{n}^{k+\frac{i}{M}}}, n_{i}^{k+\frac{i}{M}}-n_{i}^{k+\frac{i-1}{M}}\right) \geq f_{0}^{\text {ideal }}\left(\mathbf{n}^{k+\frac{i}{M}}\right)-f_{0}^{\text {ideal }}\left(\mathbf{n}^{k+\frac{i-1}{M}}\right) .
$$

Similarly, $f_{0}^{\mathrm{b}}(\mathbf{n})$ is convex with respect to $n_{i}$, and therefore

$$
\left(\left.\frac{\partial f_{0}^{\mathrm{b}}}{\partial n_{i}}\right|_{\mathbf{n}=\mathbf{n}^{k+\frac{i}{M}}}, n_{i}^{k+\frac{i}{M}}-n_{i}^{k+\frac{i-1}{M}}\right) \geq f_{0}^{\mathrm{b}}\left(\mathbf{n}^{k+\frac{i}{M}}\right)-f_{0}^{\mathrm{b}}\left(\mathbf{n}^{k+\frac{i-1}{M}}\right) .
$$

$f_{0}^{\mathrm{a}}$ is concave with respect to $n_{i}$, so

$$
\left(\left.\frac{\partial f_{0}^{\mathrm{a}}}{\partial n_{i}}\right|_{\mathbf{n}=\mathbf{n}^{k+\frac{i-1}{M}}}, n_{i}^{k+\frac{i}{M}}-n_{i}^{k+\frac{i-1}{M}}\right) \geq f_{0}^{\mathrm{a}}\left(\mathbf{n}^{k+\frac{i}{M}}\right)-f_{0}^{\mathrm{a}}\left(\mathbf{n}^{k+\frac{i-1}{M}}\right) .
$$

Copyright $@$ by SIAM. Unauthorized reproduction of this article is prohibited. 
Combining the inequalities (3.17)-(C.4), we finally conclude that

$$
\begin{aligned}
0 \geq & f_{0}\left(\mathbf{n}^{k+\frac{i}{M}}\right)+\frac{1}{2} \sum_{j, l=1}^{M} c_{j l} \nabla n_{j}^{k+\frac{i}{M}} \cdot \nabla n_{l}^{k+\frac{i}{M}} \\
& -\left(f_{0}\left(\mathbf{n}^{k+\frac{i-1}{M}}\right)+\frac{1}{2} \sum_{j, l=1}^{M} c_{j l} \nabla n_{j}^{k+\frac{i-1}{M}} \cdot \nabla n_{l}^{k+\frac{i-1}{M}}\right) \\
= & f\left(\mathbf{n}^{k+\frac{i}{M}}\right)-f\left(\mathbf{n}^{k+\frac{i-1}{M}}\right) .
\end{aligned}
$$

Integration of (C.5) yields

$$
F\left(\mathbf{n}^{k+\frac{i}{M}}\right) \leq F\left(\mathbf{n}^{k+\frac{i-1}{M}}\right)
$$

This completes the proof.

Acknowledgment. We thank the anonymous referees for their very insightful remarks and very helpful suggestions.

\section{REFERENCES}

[1] R. Amin And T. N. Smith, Interfacial tension and spreading coefficient under reservoir conditions, Fluid Phase Equilibr., 142 (1998), pp. 231-241, https://doi.org/10.1016/ S0378-3812(97)00213-6.

[2] D. M. Anderson, G. B. McFadden, and A. A. Wheeler, Diffuse-interface methods in fluid mechanics, in Annual Review of Fluid Mechanics, Annu. Rev. Fluid Mech. 30, Annual Reviews, Palo Alto, CA, 1998, pp. 139-165, https://doi.org/10.1146/annurev.fluid.30.1. 139.

[3] K. Bao, Y. Shi, S. Sun, And X. Wang, A finite element method for the numerical solution of the coupled Cahn-Hilliard and Navier-Stokes system for moving contact line problems, J. Comput. Phys., 231 (2012), pp. 8083-8099, https://doi.org/10.1016/j.jcp.2012.07.027.

[4] F. Boyer and S. Minjeaud, Numerical schemes for a three component Cahn-Hilliard model, ESAIM Math. Model. Numer. Anal., 45 (2011), pp. 697-738, https://doi.org/10.1051/ $\mathrm{m} 2 \mathrm{an} / 2010072$.

[5] B. Breure And C. J. Peters, Modeling of the surface tension of pure components and mixtures using the density gradient theory combined with a theoretically derived influence parameter correlation, Fluid Phase Equilibr., 334 (2012), pp. 189-196, https://doi.org/10.1016/j.fluid. 2012.08.010.

[6] F. Brezzi And M. Fortin, Mixed and Hybrid Finite Element Methods, Springer-Verlag, New York, 1991.

[7] C. Dawson, S. Sun, And M. F. Wheeler, Compatible algorithms for coupled flow and transport, Comput. Methods Appl. Mech. Engrg., 193 (2004), pp. 2565-2580, https: //doi.org/10.1016/j.cma.2003.12.059.

[8] V. J. Ervin, E. W. Jenkins, And S. Sun, Coupled generalized non-linear Stokes flow with flow through a porous medium, SIAM J. Numer. Anal., 47 (2009), pp. 929-952, https: //doi.org/10.1137/070708354.

[9] D. J. Eyre, An Unconditionally Stable One-Step Scheme for Gradient Systems, www.math. utah.edu/ eyre/research/methods/stable.ps?.

[10] X. FAn, J. Kou, Z. QIAO, AND S. Sun, Modeling pore-scale oil-gas systems using gradient theory with Peng-Robinson equation of state, Procedia Comput. Sci., 80 (2016), pp. 1364-1373, https://doi.org/10.1016/j.procs.2016.05.434.

[11] A. Firoozabadi, Thermodynamics of Hydrocarbon Reservoirs, McGraw-Hill, New York, 1999.

[12] T. L. Hill, Derivation of the complete van der Waals' equation from statistical mechanics, J. Chem. Educ., 25 (1948), pp. 347-348, https://doi.org/10.1021/ed025p347.

[13] J. KIM, Phase-field models for multi-component fluid flows, Commun. Comput. Phys., 12 (2012), pp. 613-661, https://doi.org/10.4208/cicp.301110.040811a.

[14] J. Kim, K. Kang, And J. Lowengrub, Conservative multigrid methods for ternary CahnHilliard systems, Commun. Math. Sci., 2 (2004), pp. 53-77, https://doi.org/10.1016/j.jcp. 2003.07.035.

Copyright (c) by SIAM. Unauthorized reproduction of this article is prohibited. 
[15] J. Kou AND S. Sun, An adaptive finite element method for simulating surface tension with the gradient theory of fluid interfaces, J. Comput. Appl. Math., 255 (2014), pp. 593-604, https://doi.org/10.1016/j.cam.2013.06.017.

[16] J. Kou AND S. Sun, Numerical methods for a multicomponent two-phase interface model with geometric mean influence parameters, SIAM J. Sci. Comput., 37 (2015), pp. B543-B569, https://doi.org/10.1137/140969579.

[17] J. Kou AND S. Sun, Unconditionally stable methods for simulating multi-component two-phase interface models with Peng-Robinson equation of state and various boundary conditions, J. Comput. Appl. Math., 291 (2016), pp. 158-182, https://doi.org/10.1016/j.cam.2015.02. 037.

[18] J. Kou And S. Sun, Multi-scale diffuse interface modeling of multi-component two-phase flow with partial miscibility, J. Comput. Phys., 318 (2016), pp. 349-372, https://doi.org/10. 1016/j.jcp.2016.04.055.

[19] J. Kou, S. Sun, AND X. WANG, Efficient numerical methods for simulating surface tension of multi-component mixtures with the gradient theory of fluid interfaces, Comput. Methods Appl. Mech. Engrg., 292 (2015), pp. 92-106, https://doi.org/10.1016/j.cma.2014.10.023.

[20] J. Kou, S. Sun, AND X. WANG, An energy stable evolutional method for simulating two-phase equilibria of multi-component fluids at constant moles, volume and temperature, Comput. Geosci., 20 (2016), pp. 283-295, https://doi.org/10.1007/s10596-016-9564-5.

[21] C. Miqueu, B. Mendiboure, A. Graciaa, and J. Lachaise, Modelling of the surface tension of pure components with the gradient theory of fluid interfaces: A simple and accurate expression for the influence parameters, Fluid Phase Equilibr., 207 (2003), pp. 225-246, https://doi.org/10.1016/S0378-3812(03)00028-1.

[22] J. Moortgat, S. Sun, And A. Firoozabadi, Compositional modeling of three-phase flow with gravity using higher-order finite element methods, Water Resour. Res., 47 (2011), W05511, http://www.agu.org/pubs/crossref/2011/2010WR009801.shtml.

[23] D. Y. Peng and D. B. Robinson, A new two-constant equation of state, Ind. Eng. Chem. Fundam., 15 (1976), pp. 59-64, https://doi.org/10.1021/i160057a011.

[24] Z. QIAO AND S. Sun, Two-phase fluid simulation using a diffuse interface model with PengRobinson equation of state, SIAM J. Sci. Comput., 36 (2014), pp. B708-B728, https: //doi.org/10.1137/130933745.

[25] Z. QIAO, Z. Sun, AND Z. Zhang, Stability and convergence of second-order schemes for the nonlinear epitaxial growth model without slope selection, Math. Comp., 84 (2015), pp. 653-674, https://doi.org/10.1090/S0025-5718-2014-02874-3.

[26] Z. QiaO, Z. Zhang, And T. TANG, An adaptive time-stepping strategy for the molecular beam epitaxy models, SIAM J. Sci. Comput., 33 (2011), pp. 1395-1414, https://doi.org/10.1137/ 100812781.

[27] L. Rongy, K. B. Haugen, and A. Firoozabadi, Mixing from Fickian diffusion and natural convection in binary non-equilibrium fluid phases, AIChE J., 58 (2012), pp. 1336-1345, https://doi.org/10.1002/aic.12685.

[28] J. Shen AND X. YAng, Decoupled energy stable schemes for phase-field models of two-phase complex fluids, SIAM J. Sci. Comput., 36 (2014), pp. B122-B145, https://doi.org/10.1137/ 130921593.

[29] S. Sun AND J. Geiser, Multiscale discontinuous Galerkin methods for modeling flow and transport in porous media, in Computational Science (ICCS 2007), Lecture Notes in Comput. Sci. 4487, Y. Shi, G. van Albada, J. Dongarra, and P. M. A. Sloot, eds., Springer, Berlin, Heidelberg, 2007, pp. 890-897, https://doi.org/10.1007/978-3-540-72584-8_117.

[30] S. Sun AND M. F. WheEler, Symmetric and nonsymmetric discontinuous Galerkin methods for reactive transport in porous media, SIAM J. Numer. Anal., 43 (2005), pp. 195-219, https://doi.org/10.1137/S003614290241708X.

[31] S. Sun AND M. F. Wheeler, Anisotropic and dynamic mesh adaptation for discontinuous Galerkin methods applied to reactive transport, Comput. Methods Appl. Mech. Engrg., 195 (2006), pp. 3382-3405, https://dx.doi.org/10.1016/j.cma.2005.06.019.

[32] S. Sun AND M. F. WheEler, Local problem-based a posteriori error estimators for discontinuous Galerkin approximations of reactive transport, Comput. Geosci., 11 (2007), pp. 87-101, https://doi.org/10.1007/s10596-007-9041-2.

[33] S. Sun AND M.F. WheELER, Discontinuous Galerkin methods for simulating bioreactive transport of viruses in porous media, Adv. Water Resour., 30 (2007), pp. 1696-1710, https://doi.org/10.1016/j.advwatres.2006.05.033.

[34] Z. Zhang AND Z. QIAO, An adaptive time-stepping strategy for the Cahn-Hilliard equation, Commun. Comput. Phys., 11 (2012), pp. 1261-1278, https://doi.org/10.4208/cicp.300810. 140411s.

Copyright $@$ by SIAM. Unauthorized reproduction of this article is prohibited. 